\title{
SOME PECULIARITIES OF APPROXIMATION METHODS FOR SINGULAR INTEGRAL EQUATIONS WITH CONJUGATION*
}

\author{
V. D. DIDENKO ${ }^{\dagger}$, S. ROCH ${ }^{\ddagger}$, AND B. SILBERMANN $\S$
}

\begin{abstract}
Approximation methods for singular integral operators with continuous coefficients and conjugation on curves with corners are investigated with respect to their stability. Particular emphasis is devoted to index constraints for the local stability conditions. It turns out that, if an associated local operator is Fredholm, then the absolute value of its Fredholm index is necessarily bounded by 2 , and this maximal value is attained in some instances (whereas it is known that in the case of pure singular integral equations Fredholmness of the associated local operators already implies vanishing index).
\end{abstract}

1. Introduction. Many problems in mathematical physics can be described by singular integral equations which contain both the unknown function and its complex conjugate, see, e.g., $[10,11,16,17,15,23]$. The presence of the complex conjugation leads to numerous new effects which have no counterparts for equations without conjugation. Concerning the applicability of certain approximation methods, some of these effects have been observed in $[8,9]$ for the equation

$$
\begin{aligned}
(A u)(t) \equiv & a(t) u(t)+\frac{b(t)}{\pi i} \int_{\Gamma} \frac{u(\tau) d \tau}{\tau-t}+c(t) \overline{u(t)}+\frac{d(t)}{\pi i} \int_{\Gamma} \overline{\frac{u(\tau)}{\tau-t}} d \tau \\
& -\frac{e(t)}{\pi i} \overline{\int_{\Gamma} \frac{u(\tau) d \tau}{\tau-t}}-\frac{r(t)}{\pi i} \overline{\int_{\Gamma} \overline{\frac{u(\tau)}{\tau-t}}} \\
& +\int_{\Gamma} k_{1}(t, \tau) u(\tau) d \tau+\int_{\Gamma} k_{2}(t, \tau) \overline{u(\tau)} d \tau=f(t), \quad t \in \Gamma
\end{aligned}
$$

where $a, b, c, d, e, f, g, k_{1}, k_{2}$ are given continuous functions, $\Gamma$ is an open or closed piecewise smooth curve in the complex plane $\mathbb{C}$, and where the operator $A$ is considered as acting on a weighted $L^{2}$-space. The double layer potential equation

$$
a(t) u(t)+\frac{b(t)}{\pi} \int_{\Gamma} u(\tau) \frac{d}{d n_{\tau}} \log |t-\tau| d \Gamma_{\tau}+(T u)(t)=f(t), \quad t \in \Gamma,
$$

where $T$ is a compact operator and $n_{\tau}$ refers to the inner normal to $\Gamma$ at the point $\tau$ is a prominent example of equations of the form (1.1) (set $c=d=e=0$ and $b=r$ in $(1.1))$. In the present paper we consider another special case of (1.1) where we

\footnotetext{
${ }^{*}$ Received Jan. 12, 1999; revised Sept. 3, 1999.

tUniversity of Brunei Darussalam, Department of Mathematics, Bandar Seri Begawan 2028, Brunei (victor@fos.ubd.edu.bn). The work of the first author was partially supported by the German Academic Exchange Service (DAAD) and by the German Research Foundation (Deutsche Forschungsgemeinschaft).

‡Fachbereich Mathematik, Technische Universität Darmstadt, Schlossgartenstrasse 7, D 64289 Darmstadt, Germany (roch@mathematik.tu-darmstadt.de).

$\S$ Fakultät für Mathematik, Technische Universität Chemnitz, D 09107 Chemnitz, Germany (bernd.silbermann@mathematik.tu-chemnitz.de).
} 
suppose the coefficients $e$ and $r$ to be identically equal to zero on $\Gamma$ :

$$
\begin{aligned}
(A u)(t) \equiv & a(t) u(t)+\frac{b(t)}{\pi i} \int_{\Gamma} \frac{u(\tau) d \tau}{\tau-t}+c(t) \overline{u(t)}+\frac{d(t)}{\pi i} \int_{\Gamma} \frac{\overline{u(\tau)} d \tau}{\tau-t} \\
& +\int_{\Gamma} k_{1}(t, \tau) u(\tau) d \tau+\int_{\Gamma} k_{2}(t, \tau) \overline{u(\tau)} d \tau=f(t), \quad t \in \Gamma .
\end{aligned}
$$

We start with describing a class of approximation methods for equation (1.3) and with establishing the corresponding stability criteria which proceeds in complete analogy to $[9,14]$. For the stability condition, we have to associate with every concrete approximation method for (1.3) (which will be specified later on), and with every point $\tau$ of $\Gamma$, a certain "model" operator $A_{\tau}$ which reflects the local behaviour of the sequence of the approximation operators at the point $\tau$. Then the stability criterion says that the approximation method is stable if and only if the operator $A$ as well as all operators $A_{\tau}$ are invertible.

The invertibility of the local operators $A_{\tau}$ (as well as that of the operator $A$ itself) can be checked effectively only in a few rare situations. However, which will be done in the present paper, the Fredholm properties of these operators, and the behaviour of their Fredholm indices, can be characterized completely. Analogous problems were studied for the first time in [9] where the basic space is $L^{2}(\Gamma)$ without weight. Particularly, we observed in [9] that the indices $\kappa\left(A_{\tau}\right)$ of the operators $A_{\tau}$ can be different from zero if $\tau$ is a corner point of $\Gamma$, but that in any case the inequality

$$
\left|\kappa\left(A_{\tau}\right)\right| \leq 1
$$

holds. The fact that the indices can be different from zero has no counterpart for singular integral equations without conjugation where it is known that the Fredholmness of the operators $A_{\tau}$ is independent of the angle at $\tau$, and that it automatically implies the vanishing of the index of $A_{\tau}, \mathrm{cf}$. $[19,20]$. Moreover, the latter result remains valid for singular integral operators without conjugation acting on $L^{2}$-spaces with power weight.

An essential change of the Fredholm properties can be observed for the local operators $A_{\tau}$ which correspond to equation (1.3) on weighted spaces. In this case, we will show that the indices of $A_{\tau}$ will not only depend on the values of the angles, but also on the weight function at the point $\tau \in \Gamma$. More precisely, if the angle $\omega_{\tau}$ of $\Gamma$ at $\tau$ belongs to one of the intervals $(0, \pi / 2]$ or $[3 \pi / 2,2 \pi)$, then the index of the corresponding operator $A_{\tau}$ is subject to the estimate (1.4) for all possible weight functions. However, if $\omega_{\tau} \in(\pi / 2, \pi) \cup(\pi, 3 \pi / 2)$, then

$$
\left|\kappa\left(A_{\tau}\right)\right| \leq 2 \text {, }
$$

and the equality sign in (1.5) holds for some combinations of coefficients, weights and angles.

We proceed with describing necessary and sufficient conditions which guarantee that the weight function can be specified in such a way that the index of every operator $A_{\tau}$ becomes equal to zero in the corresponding weighted space. The case ind $A_{\tau}=0$ for all $\tau$ is of particular interest because it allows to apply certain cutting off techniques which regularize the approximation systems in such a way that the sequence of the regularized systems becomes stable. The applicability of cutting off techniques is discussed in the concluding Section 5 . 
The case where the indices of some of the local operators are non-zero is obviously unacceptable for practically relevant approximation methods. On the other hand, the estimate (1.5) shows that the Fredholm properties of the local operators cannot be arbitrarily bad. So there is some hope that index cancellation techniques could help to manage also these situations. But this is beyond the scope of this paper.

We will illustrate the mentioned pecularities only for one special, quadrature formula based, approximation method for solving (1.3). But clearly, analogous effects appear, and can be studied in a similar manner, for other discretizations of (1.3) as well. Spline collocation and qualocation methods can serve as further examples (compare [9]). In this sense, we would like to consider this paper as a case study which can help to understand some peculiarities of approximation methods for singular integral operators with conjugation.

Throughout this paper, let $\mathcal{L}_{\text {add }}(X)$ stand for the set of all additive (i.e. $A\left(x_{1}+\right.$ $\left.x_{2}\right)=A x_{1}+A x_{2}$ for $\left.x_{1}, x_{2} \in X\right)$ and continuous operators acting on some Banach space $X$.

2. A quadrature method and its stability. Let $\Gamma$ be a simple closed curve, and let $\gamma: \mathbb{R} \rightarrow \Gamma$ be a 1-periodic parametrization of $\Gamma$. We suppose that there are points $u_{1}<u_{2}<\ldots<u_{n_{0}}=u_{1}+1$ such that $\gamma$ is twice continuously differentiable on each of the intervals $\left(u_{j}, u_{j+1}\right), j=1,2, \ldots, n_{0}-1$, and that the derivatives $\gamma^{\prime}$ and $\gamma^{\prime \prime}$ possess finite one-sided limits $\gamma^{\prime}\left(u_{j} \pm 0\right)$ and $\gamma^{\prime \prime}\left(u_{j} \pm 0\right)$ such that $\left|\gamma^{\prime}\left(u_{j}-0\right)\right|=$ $\left|\gamma^{\prime}\left(u_{j}+0\right)\right|$, but, possibly, $\arg \gamma^{\prime}\left(u_{j}-0\right) \neq \arg \gamma^{\prime}\left(u_{j}+0\right)$ at every point $u_{j}, j=$ $1, \ldots, n_{0}-1$. By $\omega=\omega(t)$ we denote the function

$$
\omega(t):=\prod_{j=1}^{n_{0}-1}\left|t-\gamma\left(u_{j}\right)\right|^{-\rho_{j}}, \quad t \in \Gamma,
$$

with $\rho_{j} \in(-1 / 2,1 / 2)$ for all $j$. Under these conditions, the operator $A$ of (1.3) acts boundedly on the Lebesgue space $L_{\omega}^{2}(\Gamma)$ of all complex-valued measurable functions $x$ with

$$
\|x\|_{L_{\omega}^{2}}:=\left(\int_{\Gamma}|x(t)|^{2} \omega^{2}(t) \mathrm{d} t\right)^{1 / 2}<\infty
$$

which is considered as a Banach space over the field $\mathbb{R}$.

To improve the convergence rate we consider discretizations based on graded meshes. For, given $\alpha \geq 1$, choose a 1-periodic function $g: \mathbb{R} \rightarrow \mathbb{R}$ which satisfies the following conditions:

1) $g\left(j / n_{0}\right)=u_{j}$ for all $j=1,2, \ldots, n_{0}-1$,

2) $g(s)=g\left(j / n_{0}\right)+\left|s-j / n_{0}\right|^{\alpha} \operatorname{sign}\left(s-j / n_{0}\right)$ for all $s$ in some neighbourhood $U_{s_{j}}$ of the point $s_{j}=j / n_{0}$, with $U_{s_{j}} \cap U_{s_{k}}=\emptyset$ if $j \neq k$, and

3 ) the function $g$ is strictly monotonically increasing on [0, 1], and its restriction onto the set $\left[s_{j}, s_{j+1}\right] \backslash\left(U_{s_{j}} \cap U_{s_{j+1}}\right)$ is a twice differentiable function for every $j$.

Let $n=\ln _{0}$ with $l \in \mathbb{N}$, choose numbers $\delta, \varepsilon \in(0,1)$ with $\delta \neq \varepsilon$, define $\tilde{\gamma}(s)=$ $\gamma(g(s))$, and for each $k=0,1, \ldots, n-1$, put

$$
t_{k}^{(n)}:=\tilde{\gamma}\left(\frac{k+\delta}{n}\right), \quad \tau_{k}^{(n)}:=\tilde{\gamma}\left(\frac{k+\varepsilon}{n}\right), \quad \Delta t_{j}^{(n)}=\frac{1}{n} \tilde{\gamma}\left(\frac{j+\delta}{n}\right) .
$$


Applying a shifted trapezoidal rule to the singular integrals in (1.3) and then collocating at the differently shifted points $\tau_{k}^{(n)}$ leads to the following system of algebraic equations

$$
\begin{aligned}
& {\left[a\left(\tau_{k}^{(n)}\right)-b\left(\tau_{k}^{(n)}\right) i \cot (\pi(\varepsilon-\delta))\right] \xi_{k}^{(n)}+\frac{b\left(\tau_{k}^{(n)}\right)}{\pi i} \sum_{j=0}^{n-1} \frac{\Delta t_{j}^{(n)}}{t_{j}^{(n)}-\tau_{k}^{(n)}} \xi_{j}^{(n)}} \\
& +\left[c\left(\tau_{k}^{(n)}\right)-d\left(\tau_{k}^{(n)}\right) i \cot (\pi(\varepsilon-\delta))\right] \overline{\xi_{k}^{(n)}}+\frac{d\left(\tau_{k}^{(n)}\right)}{\pi i} \sum_{j=0}^{n-1} \frac{\Delta t_{j}^{(n)}}{t_{j}^{(n)}-\tau_{k}^{(n)}} \overline{\xi_{j}^{(n)}} \\
& +\sum_{j=0}^{n-1} k_{1}\left(t_{k}^{(n)}, t_{j}^{(n)}\right) \Delta t_{j}^{(n)} \xi_{j}^{(n)}+\sum_{j=0}^{n-1} k_{2}\left(t_{k}^{(n)}, t_{j}^{(n)}\right) \Delta t_{j}^{(n)} \overline{\xi_{j}^{(n)}} \\
& \quad=f\left(\tau_{k}^{(n)}\right), \quad k=0,1, \cdots, n-1,
\end{aligned}
$$

where the $\xi_{k}^{(n)}$ are thought of as approximations of the values of the solution $u$ of (1.3) at the points $t_{k}^{(n)}$ (for details we refer to $[9,14,20]$ ). If $\chi_{j}^{(n)}(t)$ denotes the characteristic function of the subarc $[\tilde{\gamma}(j / n), \tilde{\gamma}((j+1) / n))$ of $\Gamma$, then the function

$$
x_{n}(t)=\sum_{j=0}^{n-1} \xi_{j}^{(n)} \chi_{j}^{(n)}(t), \quad t \in \Gamma .
$$

can be viewed as an approximate solution of equation (1.3).

The stability of the approximation method (2.2)-(2.3) can be studied by means of localizing techniques as in $[9,14,20]$. For, we associate with every point $\tau \in \Gamma$ a model equation $A^{\tau} x^{\tau}=f^{\tau}$, which locally represents the equation (1.3), as well as a model approximation method for the model equation

$$
A_{n}^{\tau} x_{n}^{\tau}=f_{n}^{\tau},
$$

which locally represents (2.2). This association can be done as follows: Given $\tau \in \Gamma$, let $s_{\tau}$ refer to that point of $[0,1)$ with $\tilde{\gamma}\left(s_{\tau}\right)=\tau$, denote by $\omega_{\tau}$ the number

$$
\omega_{\tau}:=\arg \left(-\tilde{\gamma}^{\prime}\left(s_{\tau}-0\right) / \tilde{\gamma}^{\prime}\left(s_{\tau}+0\right)\right), \quad \omega_{\tau} \in[0,2 \pi),
$$

and write $\Gamma_{\tau}$ for the angle $\Gamma_{\tau}:=\mathrm{e}^{\mathrm{i} \omega_{\tau}}[0,+\infty) \cup[0,+\infty)$ with the ray $\mathrm{e}^{\mathrm{i} \omega_{\tau}}[0,+\infty)$ being directed to the origin, while $[0,+\infty)$ is directed away from it. Then define $L^{2}\left(\Gamma_{\tau}, \rho_{\tau}\right)$ as the real Banach space of all complex-valued Lebesgue measurable functions on $\Gamma_{\tau}$ such that

$$
\int_{\Gamma_{\tau}}|x(t)|^{2}|t|^{-2 \rho_{\tau}}(t) \mathrm{d} t<+\infty
$$

where $\rho_{\tau}=\rho_{j}$ if $\tau=\gamma\left(u_{j}\right)\left(j=1,2, \ldots, n_{0}-1\right)$ and $\rho_{\tau}=0$ at all other points $\tau$. Further introduce the operators

$$
\left(S_{\Gamma_{\tau}} x\right)(t):=\frac{1}{\pi \mathrm{i}} \int_{\Gamma_{\tau}} \frac{x(u) \mathrm{d} u}{u-t}, \quad(M x)(t):=\overline{x(t)}, \quad t \in \Gamma_{\tau},
$$

as well as

$$
A^{\tau}:=a(\tau) I+b(\tau) S_{\Gamma_{\tau}}+c(\tau) M+d(\tau) S_{\Gamma_{\tau}} M
$$


which act boundedly on $L^{2}\left(\Gamma_{\tau}, \rho_{\tau}\right)$. Now, the model equation has the form

$$
A^{\tau} x^{\tau}=f^{\tau}, \quad x^{\tau} \in L^{2}\left(\Gamma_{\tau}, \rho_{\tau}\right),
$$

with a function $f^{\tau}$ belonging to the Banach space $R^{2}\left(\Gamma_{\tau}, \rho_{\tau}\right)$ of all functions which are Riemann integrable on every finite subcurve of $\Gamma_{\tau}$ and have a finite Riemann norm

$$
\begin{aligned}
\|f\|_{R^{2}\left(\Gamma_{\tau}, \rho_{\tau}\right)}:= & \|f\|_{L^{2}\left(\Gamma_{\tau}, \rho_{\tau}\right)}+\left(\sum_{k=0}^{\infty} \sup _{t \in[k, k+1]}|f(t)|^{2}|t|^{-2 \rho_{\tau}}\right)^{1 / 2} \\
& +\left(\sum_{k=0}^{\infty} \sup _{t \in e^{i \omega_{\tau}[k, k+1]}}|f(t)|^{2}|t|^{-2 \rho_{\tau}}\right)^{1 / 2} .
\end{aligned}
$$

For the approximate solution of equation (2.6), introduce points $\tilde{t}_{k}^{(n)}$ and $\tilde{\tau}_{k}^{(n)}$, $k \in \mathbb{Z}$, by

$$
\tilde{t}_{k}^{(n)}:=\left\{\begin{array}{cl}
\left(\frac{k+\delta}{n}\right)^{\alpha} & \text { if } k \geq 0 \\
-\left(\frac{k+\delta}{n}\right)^{\alpha} e^{i \omega_{\tau}} & \text { if } k<0
\end{array}, \quad \tilde{\tau}_{k}^{(n)} \quad:=\left\{\begin{array}{cl}
\left(\frac{k+\varepsilon}{n}\right)^{\alpha} & \text { if } k \geq 0 \\
-\left(\frac{k+\varepsilon}{n}\right)^{\alpha} e^{i \omega_{\tau}} & \text { if } \quad k<0
\end{array},\right.\right.
$$

and determine approximate values $\xi_{k}^{(n)}$ of the exact solution $x^{\tau}\left(\tilde{t}_{k}^{(n)}\right), k \in \mathbb{Z}$, of (2.6) by solving the infinite model system

$$
\begin{aligned}
& {[a(\tau)-b(\tau) i \cot (\pi(\varepsilon-\delta))] \xi_{k}^{(n)}+\frac{b(\tau)}{\pi i} \sum_{j=-\infty}^{+\infty} \frac{\Delta \tilde{t}_{j}^{(n)}}{\tilde{t}_{j}^{(n)}-\tilde{\tau}_{k}^{(n)}} \xi_{j}^{(n)}} \\
& +[c(\tau)-d(\tau) i \cot (\pi(\varepsilon-\delta))] \overline{\xi_{k}^{(n)}}+\frac{d(\tau)}{\pi i} \sum_{j=-\infty}^{+\infty} \frac{\Delta \tilde{t}_{j}^{(n)}}{\tilde{t}_{j}^{(n)}-\tilde{\tau}_{k}^{(n)}} \overline{\xi_{j}^{(n)}} \\
& \quad=f\left(\tilde{\tau}_{k}^{(n)}\right) \quad \text { for } k \in \mathbb{Z}
\end{aligned}
$$

where

$$
\Delta \tilde{t}_{j}^{(n)}=\left\{\begin{array}{cc}
\frac{\alpha}{n} \cdot\left(\frac{j+\delta}{n}\right)^{\alpha-1} & \text { if } j \geq 0 \\
-\frac{\alpha}{n} \cdot\left(\frac{j+\delta}{n}\right)^{\alpha-1} e^{i \omega_{\tau}} & \text { if } \quad j<0
\end{array} .\right.
$$

If $\tilde{\chi}_{k}^{(n)}$ stands for the characteristic function of the interval $\left[(k / n)^{\alpha},((k+1) / n)^{\alpha}\right]$ in case $k \geq 0$ and of the interval $\left[e^{i \omega_{\tau}}(k / n)^{\alpha}, e^{i \omega_{\tau}}((k+1) / n)^{\alpha}\right]$ in case $k<0$, then the approximate solution of (2.6) on the whole curve $\Gamma_{\tau}$ is given by

$$
x_{n}^{\tau}(t)=\sum_{k \in \mathbb{Z}} \xi_{k}^{(n)} \tilde{\chi}_{k}^{(n)}(t), \quad t \in \Gamma_{\tau} .
$$

One can think of the infinite model system (2.7) - (2.8) as an operator equation

$$
A_{n}^{\tau} x_{n}^{\tau}=f_{n}^{\tau}
$$

with $x_{n}^{\tau}, f_{n}^{\tau} \in L^{2}\left(\Gamma_{\tau}, \rho_{\tau}\right)$ and $A_{n}^{\tau} \in \mathcal{L}_{a d d}\left(L^{2}\left(\Gamma_{\tau}, \rho_{\tau}\right)\right)$. Note that the boundedness of $A_{n}^{\tau}$ follows from the corresponding results of [14], Chapters 2 and 3 , or of [19]. But 
it is more convenient for our purposes to translate this system into an equation on appropriately chosen little $l^{2}$-spaces.

Let $\tilde{l}_{\nu}^{2}, \nu \in \mathbb{R}$, be the Banach space of all two-sided sequences $\left\{\xi_{k}\right\}_{k \in \mathbb{Z}}$ of complex numbers such that

$$
\left\|\left\{\xi_{k}\right\}_{k \in \mathbb{Z}}\right\|_{\tilde{i}_{\nu}^{2}}:=\left(\sum_{k \in \mathbb{Z}}\left|\xi_{k}\right|^{2}(|k|+1)^{2 \nu}\right)^{1 / 2}<\infty .
$$

Set $r_{\tau}:=(\alpha-1) / 2-\rho_{\tau} \alpha$. It is well known (see, e.g., $\left.[14,20]\right)$ that there is a constant $c>0$ such that

$$
\frac{1}{c}\left\|\sum_{k \in \mathbb{Z}} \xi_{k} \tilde{\chi}_{k}^{(n)}\right\|_{L^{2}\left(\Gamma_{\tau}, \rho_{\tau}\right)} \leq n^{-r_{\tau}-1 / 2}\left\|\left\{\xi_{k}\right\}_{k \in \mathbb{Z}}\right\|_{\tilde{l}_{r_{\tau}}^{2}} \leq c\left\|\sum_{k \in \mathbb{Z}} \xi_{k} \tilde{\chi}_{k}^{(n)}\right\|_{L^{2}\left(\Gamma_{\tau}, \rho_{\tau}\right)}
$$

for every sequence $\left\{\xi_{k}\right\} \in \tilde{l}_{r_{\tau}}^{2}$. The de Boor inequalities (2.10) allow to identify the system (2.7) with an operator equation

$$
\tilde{A}_{n}^{\tau} \xi_{n}=\eta_{n}
$$

where $\xi_{n}=\left\{\xi_{k}^{(n)}\right\}_{k \in \mathbb{Z}}$ and $\eta_{n}=\left\{f\left(\tilde{\tau}_{k}^{(n)}\right)\right\}_{k \in \mathbb{Z}}$ belong to $\tilde{l}_{r_{\tau}}^{2}$ and $\tilde{A}_{n}^{\tau} \in \mathcal{L}_{a d d}\left(\tilde{l}_{r_{\tau}}^{2}\right)$. Moreover, again due to (2.10), the sequence $\left\{A_{n}^{\tau}\right\}_{n \in \mathbb{N}}$ in (2.9) is stable if and only if the sequence $\left\{\tilde{A}_{n}^{\tau}\right\}_{n \in \mathbb{N}}$ in (2.11) is so. Now the advantage of considering the model problem on $\tilde{l}_{r_{\tau}}^{2}$ becomes obvious: Namely, the operators $\tilde{A}_{n}^{\tau}, n \in \mathbb{N}$ turn out to be independent of $n$, thus, the sequence $\left\{\tilde{A}_{n}^{\tau}\right\}_{n \in \mathbb{N}}$ is actually constant, and so this sequence is stable if and only if one of its elements, say the operator $\tilde{A}_{1}^{\tau}$, is invertible.

Applying the local principle analogously to $[9,14]$ one arrives at the following result.

TheOREM 2.1. Let $a, b, c, d \in C(\Gamma)$, and let $k_{1}, k_{2} \in C(\Gamma \times \Gamma)$. The quadrature method (2.2) - (2.3) is stable if and only if the operator $A \in \mathcal{L}_{a d d}\left(L^{2}\left(\Gamma_{\tau}, \rho_{\tau}\right)\right)$ as well as all operators $\tilde{A}_{1}^{\tau} \in \mathcal{L}_{a d d}\left(\tilde{l}_{r_{\tau}}^{2}\right), \tau \in \Gamma$, are invertible.

The difference between the situation examined in [9] and the previous theorem is that what we study here is weighted spaces (i.e. $\rho \neq 0$ ) and non-uniformly graded meshes (i.e. $\alpha \neq 0$ ). These modifications do not influence seriously the proof of Theorem 2.1, but they essentially change the Fredholm properties of the $\tilde{A}_{1}^{\tau}$ as we will see now.

3. Local operators and their indices. As mentioned above, both the Fredholm properties and the index of the local operators $\tilde{A}_{1}^{\tau}$ which correspond to pure singular integral equations without conjugation are independent both of the angles $\omega_{\tau}$ and of the weights $\rho_{\tau}$ (see $[19,20]$ ). On the other hand, as we observed in [9] (where the weight function was supposed to be identically 1 ), the index of the operators $\tilde{A}_{1}^{\tau}$ which correspond to singular integral equations with conjugation can differ from zero; however, it can take the values $-1,0$ and 1 only. Now we are going to examine how the change of the weight function influences the index.

We start with representing the local operators $\tilde{A}_{1}^{\tau}$ as a $2 \times 2$ operator matrix. To this end we identify the Hilbert space $\tilde{l}_{r_{\tau}}^{2}$ with the direct sum $l_{r_{\tau}}^{2} \oplus l_{r_{\tau}}^{2}$ of the corresponding Hilbert spaces of one-sided sequences. Then every operator on $\tilde{l}_{r_{\tau}}^{2}$ corresponds to a $2 \times 2$ matrix whose entries are operators on $l_{r_{\tau}}^{2}$.

Further, given $\nu$, let $\mathcal{T}_{\nu}$ stand for the smallest closed subalgebra of $\mathcal{L}\left(l_{\nu}^{2}\right)$ which contains all Toeplitz operators generated by piecewise constant functions, and let 
$\mathcal{T}_{\nu}^{2 \times 2}$ be the subalgebra of $\mathcal{L}\left(\tilde{l}_{\nu}^{2}\right) \cong \mathcal{L}\left(l_{\nu}^{2} \oplus l_{\nu}^{2}\right) \cong \mathcal{L}^{2 \times 2}\left(l_{\nu}^{2}\right)$ which consists of all $2 \times 2$ matrices with entries in $\mathcal{T}_{\nu}$. (Recall that the Toeplitz operator $T(a)$ with generating function $a \in L^{\infty}$ is defined via its matrix representation with respect to the standard basis of $l_{\nu}^{2}$ by $T(a)=\left(a_{j-k}\right)_{j, k=0}^{\infty}$ with $a_{k}$ referring to the $k$ th Fourier coefficient of $a$. If $a$ is a piecewise constant function, then $T(a)$ is a bounded operator on $l_{\nu}^{2}$ if $-1 / 2<\nu<1 / 2$.)

LEMMA 3.1. Every operator $\tilde{A}_{1}^{\tau}$ as in (2.11) can be written as

$$
\tilde{A}_{1}^{\tau}=\tilde{A}^{1, \tau}+\tilde{A}^{2, \tau} \bar{M}
$$

where $\tilde{A}^{1, \tau}, \tilde{A}^{2, \tau} \in \mathcal{T}_{r_{\tau}}^{2 \times 2}$, and where $\bar{M}$ is the operator

$$
\bar{M}\left\{\left\{\xi_{k}\right\}_{k=0}^{\infty},\left\{\eta_{k}\right\}_{k=0}^{\infty}\right\}=\left\{\left\{\overline{\xi_{k}}\right\}_{k=0}^{\infty},\left\{\overline{\eta_{k}}\right\}_{k=0}^{\infty} .\right\}
$$

Proof. Fix a point $\tau \in \Gamma$ and let for brevity

$$
a=a(\tau), \quad b=b(\tau), \quad c=c(\tau), \quad d=d(\tau), \quad \rho=\rho_{\tau}, \quad \omega=\omega_{\tau} .
$$

Straightforward computation shows that the operator $\tilde{A}_{1}^{\tau}$ can be written in the form (3.1) with

$$
\tilde{A}^{1, \tau}=\left(A_{k, r}^{1, \tau}\right)_{k, r=1}^{2}, \quad \tilde{A}^{2, \tau}=\left(A_{k, r}^{2, \tau}\right)_{k, r=1}^{2}
$$

where

$$
\begin{gathered}
A_{1,1}^{1, \tau}=(a-\mathrm{i} b \cot (\pi(\varepsilon-\delta))) I+\frac{b}{\pi \mathrm{i}}\left(\frac{\alpha(j+\delta)^{\alpha-1}}{(j+\delta)^{\alpha}-(k+\varepsilon)^{\alpha}}\right)_{k, j=0}^{\infty} \\
A_{2,1}^{1, \tau}=\frac{b}{\pi \mathrm{i}}\left(\frac{\alpha(j+\delta)^{\alpha-1}}{(j+\delta)^{\alpha}-(k+1-\varepsilon)^{\alpha}} \mathrm{e}^{\mathrm{i} \omega}\right)_{k, j=0}^{\infty}, \\
A_{1,2}^{1, \tau}=\frac{b}{\pi \mathrm{i}}\left(\frac{\alpha(j+1-\delta)^{\alpha-1} \mathrm{e}^{\mathrm{i} \omega}}{(j+1-\delta)^{\alpha} \mathrm{e}^{\mathrm{i} \omega}-(k+\varepsilon)^{\alpha}}\right)_{k, j=0}^{\infty}, \\
A_{2,2}^{1, \tau}=(a-\mathrm{i} b \cot (\pi(\delta-\varepsilon))) I-\frac{b}{\pi \mathrm{i}}\left(\frac{\alpha(j+1-\delta)^{\alpha-1}}{(j+1-\delta)^{\alpha}-(k+1-\varepsilon)^{\alpha}}\right)_{k, j=0}^{\infty} .
\end{gathered}
$$

Replacing $a$ and $b$ by $c$ and $d$, respectively, one gets analogous representations for the operators $A_{k, r}^{2, \tau}, k, r=1,2$.

Let further $\Lambda^{z}, z \in \mathbb{C}$, refer to the operator of multiplication by the diagonal matrix $\Lambda^{z}:=\left((j+1)^{z} \delta_{j, k}\right)_{k, j=0}^{+\infty}$. Evidently, $\Lambda^{z}$ is a linear isometry from $l_{z}^{2}$ onto $l^{2}$ (where, clearly, $l^{2}=l_{0}^{2}$ ). Therefore, $\tilde{A}_{1}^{\tau}$ is a continuous additive operator on $l_{r_{\tau}}^{2} \oplus l_{r_{\tau}}^{2}$ if and only if the operator

$$
\hat{A}_{1}^{\tau}:=\left(\begin{array}{cc}
\Lambda^{r_{\tau}} & 0 \\
0 & \Lambda^{r_{\tau}}
\end{array}\right) \tilde{A}_{1}^{\tau}\left(\begin{array}{cc}
\Lambda^{-r_{\tau}} & 0 \\
0 & \Lambda^{-r_{\tau}}
\end{array}\right)
$$


belongs to $\mathcal{L}_{\text {add }}^{2 \times 2}\left(l^{2}\right)$. Since $\Lambda^{z} \bar{M}=\bar{M} \Lambda^{z}$ for every real $z$, the operator $\hat{A}_{1}^{\tau}$ can be rewritten in the form

$$
\hat{A}_{1}^{\tau}=\hat{A}^{1, \tau}+\hat{A}^{2, \tau} \bar{M}
$$

with $\hat{A}^{i, \tau}=\Lambda^{(\alpha-1) / 2-\rho \alpha} \tilde{A}^{i, \tau} \Lambda^{(-\alpha+1) / 2+\rho \alpha}$ for $i=1,2$. To complete the proof one has to check that the latter operators belong to the algebra $\mathcal{T}_{0}^{2 \times 2}$. This has actually be done in [20], pp. 46-49, and [14], Sections 2.4 and 2.11.

We further need the following result; a proof is in [9].

Lemma 3.2. (a) If $\hat{A} \in \mathcal{T}_{0}$, then $\bar{M} \hat{A} \bar{M}$ is in $\mathcal{T}_{0}$ again.

(b) The operator $\tilde{A}_{1}^{\tau}$ in (3.1) is Fredholm on the real space $\tilde{l}^{2}$ if and only if the operator

$$
A_{1, \tau}:=\left(\begin{array}{cc}
\hat{A}^{1, \tau} & \hat{A}^{2, \tau} \\
\bar{M} \hat{A}^{2, \tau} \bar{M} & \bar{M} \hat{A}^{1, \tau} \bar{M}
\end{array}\right)
$$

is Fredholm on the corresponding complex space. In that case,

$$
\operatorname{ind}_{\mathbb{C}} A_{1, \tau}=\operatorname{ind}_{\mathbb{R}} \tilde{A}_{1}^{\tau} .
$$

In what follows we will fix the point $\tau \in \Gamma$. Thus, we drop the subscript $\tau$ in $A_{1, \tau}$, but we will indicate the dependence of that operator from $\alpha, \rho$ and $\omega$ by writing $A_{1}^{\alpha, \rho, \omega}$ in place of $A_{1, \tau}$. The Fredholmness of the operator $A_{1}^{\alpha, \rho, \omega}$ can be studied via the Gohberg/Krupnik symbol calculus for the algebra $\mathcal{T}_{0}^{2 \times 2}$. For that goal, we let $f^{\nu}$ stand for the function

$$
f^{\nu}\left(\mathrm{e}^{\mathrm{i} 2 \pi s}\right)=2 \mathrm{e}^{\mathrm{i} \pi \nu(s-1)} \frac{\sin (-\pi \nu s)}{\sin (-\pi \nu)}-1 \text { for } 0 \leq s<1,
$$

and we set $f^{*}(t):=\overline{f(\bar{t})}, \beta:=\beta(\mu)=\sin (\pi \theta), \gamma:=\gamma(\mu)=-\mathrm{i} \cot (\pi \theta)$, and $\theta:=$ $\theta(\mu)=\frac{1}{2}-\rho+\frac{\mathrm{i}}{2 \pi \alpha} \log \frac{\mu}{1-\mu}, \mu \in[0,1]$. Then the $\operatorname{symbol} \operatorname{Sym}\left(A_{1}^{\alpha, \rho, \omega}\right)$ of the operator $A_{1}^{\alpha, \rho, \omega}$, which is a $4 \times 4$-matrix-valued function defined on $\mathbb{T} \times[0,1]$, is given as follows (see $[1,9,13,14,20])$ :

If $t \neq 1$ and $\mu \in[0,1]$, then $\left(\operatorname{Sym} A_{1}^{\alpha, \rho, \omega}\right)(t, \mu)$ is equal to

$$
\left(\begin{array}{cccc}
a+b f^{(\varepsilon-\delta)}(t) & 0 & c+d f^{(\varepsilon-\delta)}(t) & 0 \\
0 & a-b f^{(\delta-\varepsilon)}(t) & 0 & c-d f^{(\delta-\varepsilon)}(t) \\
\bar{c}+\bar{d} f^{(\varepsilon-\delta) *}(t) & 0 & \bar{a}+\bar{b} f^{(\varepsilon-\delta) *}(t) & 0 \\
0 & \bar{c}-\bar{d} f^{(\delta-\varepsilon) *}(t) & 0 & \bar{a}-\bar{b} f^{(\delta-\varepsilon) *}(t)
\end{array}\right)
$$

If $t=1$ and $\mu \in[0,1]$, then $\left(\operatorname{Sym} A_{1}^{\alpha, \rho, \omega}\right)(1, \mu)$ is given by

$$
\left(\begin{array}{cccc}
a+b \gamma & \mathrm{i} \frac{b}{\beta} \mathrm{e}^{\mathrm{i}(\omega-\pi) \theta} & c+d \gamma & \mathrm{i} \frac{d}{\beta} \mathrm{e}^{\mathrm{i}(\omega-\pi) \theta} \\
-\mathrm{i} \frac{b}{\beta} \mathrm{e}^{\mathrm{i}(\pi-\omega) \theta} & a-b \gamma & -\mathrm{i} \frac{d}{\beta} \mathrm{e}^{\mathrm{i}(\pi-\omega) \theta} & c-d \gamma \\
\bar{c}-\bar{d} \gamma & -\mathrm{i} \frac{\bar{d}}{\beta} \mathrm{e}^{\mathrm{i}(\pi-\omega) \theta} & \bar{a}-\bar{b} \gamma & -\mathrm{i} \frac{\bar{b}}{\beta} \mathrm{e}^{\mathrm{i}(\pi-\omega) \theta} \\
\mathrm{i} \frac{\bar{d}}{\beta} \mathrm{e}^{\mathrm{i}(\omega-\pi) \theta} & \bar{c}+\bar{d} \gamma & \mathrm{i} \frac{\bar{b}}{\beta} \mathrm{e}^{\mathrm{i}(\omega-\pi) \theta} & \bar{a}+\bar{b} \gamma
\end{array}\right)
$$

We prepare the investigation of the Fredholmness of the local operator $A_{1}^{\alpha, \rho, \omega}$ by mentioning two important properties of that operator. 
LEMMA 3.3. If $t \neq 1$, then $\operatorname{det}\left\{\operatorname{Sym} A_{1}^{\alpha, \rho, \omega}(t, \mu)\right\}$ is real and non-negative for arbitrary $\mu \in[0,1]$ and $a, b, c, d \in \mathbb{C}$.

A proof of this assertion is in our paper [9]. Here we offer another proof which is essentially simpler than the former one. Put $\nu=\varepsilon-\delta$ and multiply the matrix (3.4) both from the left and from the right hand side by the matrix

$$
J:=\left(\begin{array}{cccc}
1 & 0 & 0 & 0 \\
0 & 0 & 1 & 0 \\
0 & 1 & 0 & 0 \\
0 & 0 & 0 & 1
\end{array}\right)
$$

This transformation does not influence the determinant, and the new matrix has the form

$$
\left(\begin{array}{cccc}
a+b f^{\nu}(t) & c+d f^{\nu}(t) & 0 & 0 \\
\bar{c}+\bar{d} f^{\nu *}(t) & \bar{a}+\bar{b} f^{\nu *}(t) & 0 & 0 \\
0 & 0 & a-b f^{-\nu}(t) & c-d f^{-\nu}(t) \\
0 & 0 & \bar{c}-\bar{d} f^{-\nu *}(t) & \bar{a}-\bar{b} f^{-\nu *}(t)
\end{array}\right)
$$

Its determinant is just

$$
\begin{aligned}
& {\left[\left(a+b f^{\nu}(t)\right)\left(\bar{a}+\bar{b} f^{\nu *}(t)\right)-\left(c+d f^{\nu}(t)\right)\left(\bar{c}+\bar{d} f^{\nu *}(t)\right)\right]} \\
& \cdot\left[\left(a-b f^{-\nu}(t)\right)\left(\bar{a}-\bar{b} f^{-\nu *}(t)\right)-\left(c-d f^{-\nu}(t)\right)\left(\bar{c}-\bar{d} f^{-\nu *}(t)\right)\right]
\end{aligned}
$$

and taking into account the identities

$$
f^{\nu *}(t)=-f^{\nu}(t), \quad \overline{f^{\nu}(t)}=f^{-\nu}(t),
$$

we can write (3.6) in the form

$$
\begin{aligned}
& {\left[\left(a+b f^{\nu}(t)\right)\left(\bar{a}-\bar{b} f^{\nu}(t)\right)-\left(c+d f^{\nu}(t)\right)\left(\bar{c}-\bar{d} f^{\nu}(t)\right)\right]} \\
& \cdot\left[\overline{\left(a+b f^{\nu}(t)\right)}\left(a-b \overline{f^{\nu}(t)}\right)-\overline{\left(c+d f^{\nu}(t)\right)}\left(c+d \overline{f^{\nu}(t)}\right)\right],
\end{aligned}
$$

which is obviously real and non-negative.

Lemma 3.4. For any $\alpha_{1}, \alpha_{2} \in \mathbb{R}, \alpha_{1} \geq 1, \alpha_{2} \geq 1$, the operators $A_{1}^{\alpha_{1}, \rho, \omega}$ and $A_{1}^{\alpha_{2}, \rho, \omega}$ are simultaneously Fredholm or not. In case they are Fredholm,

$$
\text { ind } A_{1}^{\alpha_{1}, \rho, \omega}=\operatorname{ind} A_{1}^{\alpha_{2}, \rho, \omega} \text {. }
$$

Proof. A closer look at the symbol of the operator $A_{1}^{\alpha, \rho, \omega}$ reveals that only the matrix (3.5) depends on the grading parameter $\alpha$ via the function $\theta$. But the image of the function $\theta=\theta(\mu), \mu \in[0,1]$, coincides with $1 / 2-\rho+\mathrm{i} \mathbb{R}$ which is independent on $\alpha$.

So we can suppose here and hereafter that $\alpha=1$, and we will write $A_{1}^{\rho, \omega}$ in place of $A_{1}^{1, \rho, \omega}$. Recall from [9] that the index of $A_{1}^{0, \omega}$ can take the values $-1,0$ or 1 only. Our next goal is to verify that this assertion remains true for $\rho \in(0,1 / 2)$ as well. This proof is essentially different from that given in [9] for case $\rho=0$.

Lemma 3.5. The determinant of $\operatorname{Sym} A_{1}^{\rho, \omega}(1, \mu)$ can be represented as

$$
\operatorname{det}\left\{\operatorname{Sym} A_{1}^{\rho, \omega}(1, \mu)\right\}=U+V \Phi^{2}(\mu),
$$


with certain real numbers $U, V$, and with $\Phi$ denoting the function

$$
\Phi(\mu)=\Phi_{\rho, \omega}(\mu)=\frac{\sin ((\omega-\pi) \theta)}{\sin (\pi \theta)}, \quad \mu \in[0,1]
$$

where $\theta=\theta(\mu)$ is as above.

Proof. Expanding the determinant according to the second order minors of the first two rows gives

$$
\begin{aligned}
& \operatorname{det}\left\{\operatorname{Sym} A_{1}^{\rho, \omega}(1, \mu)\right\} \\
& =\left[(a+b \gamma)(a-b \gamma)-\frac{b^{2}}{\beta^{2}}\right]\left[(\bar{a}+\bar{b} \gamma)(\bar{a}-\bar{b} \gamma)-\frac{\overline{b^{2}}}{\beta^{2}}\right] \\
& \quad+\left[(c+d \gamma)(c-d \gamma)-\frac{d^{2}}{\beta^{2}}\right]\left[(\bar{c}+\bar{d} \gamma)(\bar{c}-\bar{d} \gamma)-\frac{\overline{d^{2}}}{\beta^{2}}\right] \\
& \quad+\left[(a+b \gamma)(c-d \gamma)-\frac{b d}{\beta^{2}}\right]\left[\frac{\overline{b d}}{\beta^{2}}-(\bar{a}-\bar{b} \gamma)(\bar{c}+\bar{d} \gamma)\right] \\
& \quad+\left[(\bar{a}+\bar{b} \gamma)(\bar{c}-\bar{d} \gamma)-\frac{\overline{b d}}{\beta^{2}}\right]\left[\frac{b d}{\beta^{2}}-(a-b \gamma)(c+d \gamma)\right] \\
& +\frac{1}{\beta^{2}}(a d-b c)(\overline{a d-b c}) \mathrm{e}^{\mathrm{i} 2(\pi-\omega) \theta} \\
& +\frac{1}{\beta^{2}}(a d-b c)(\overline{a d-b c}) \mathrm{e}^{\mathrm{i} 2(\omega-\pi) \theta}
\end{aligned}
$$

The first line on the right hand side of (3.8) is equal to

$$
\begin{aligned}
& {\left[a^{2}-b^{2} \gamma^{2}-\frac{b^{2}}{\beta^{2}}\right]\left[\bar{a}^{2}-\bar{b}^{2} \gamma^{2}-\frac{\overline{b^{2}}}{\beta^{2}}\right]} \\
& =\left[a^{2}-b^{2}\left(-\cot ^{2}(\pi \theta)+\frac{1}{\sin ^{2}(\pi \theta)}\right)\right]\left[\bar{a}^{2}-\bar{b}^{2}\left(-\cot ^{2}(\pi \theta)+\frac{1}{\sin ^{2}(\pi \theta)}\right)\right] \\
& =\left|a^{2}-b^{2}\right|^{2}
\end{aligned}
$$

analogously, the second one coincides with $\left|c^{2}-d^{2}\right|^{2}$. For the third line, abbreviate $a c-b d$ and $c b-a d$ by $N$ and $P$, respectively, and note that

$$
(a+b \gamma)(c-d \gamma)-\frac{b d}{\beta^{2}}=N+P \gamma, \quad \frac{\overline{b d}}{\beta^{2}}-(\bar{a}-\bar{b} \gamma)(\bar{c}+\bar{d} \gamma)=-\bar{N}+\bar{P} \gamma
$$

which finally gives $-|N|^{2}-2 \mathrm{i} \operatorname{Im}(P \bar{N}) \gamma+|P|^{2} \gamma^{2}$ for the third and, analogously, $-|N|^{2}+$ $2 \mathrm{i} \operatorname{Im}(P \bar{N}) \gamma+|P|^{2} \gamma^{2}$ for the forth line. Putting all pieces together we find

$$
\begin{aligned}
& \operatorname{det}\left\{\operatorname{Sym} A_{1}^{\rho, \omega}(1, \mu)\right\} \\
& =\left|a^{2}-b^{2}\right|^{2}+\left|c^{2}-d^{2}\right|^{2}-2|N|^{2}+2|P|^{2} \gamma^{2}+2|P| \frac{\cos (2(\omega-\pi) \theta)}{\sin ^{2}(\pi \theta)} \\
& =\left|a^{2}-b^{2}\right|^{2}+\left|c^{2}-d^{2}\right|^{2}-2|N|^{2}+2|P|^{2}\left(1-2\left(\frac{\sin ((\omega-\pi) \theta)}{\sin (\pi \theta)}\right)^{2}\right) \\
& =U+V \Phi^{2}(\mu)
\end{aligned}
$$


with

$$
V=-4|P|^{2}, \quad U=\left|a^{2}-b^{2}\right|^{2}+\left|c^{2}-d^{2}\right|^{2}+2\left(|P|^{2}-|N|^{2}\right)
$$

as desired.

CoRollary 3.6. If $c b-a d=0$ and if the operator $A_{1}^{\rho, \omega}$ is Fredholm, then its index is zero.

The condition $c b-a d=0$ is in particular true for singular integral equations without conjugation (where $c=0, d=0$ ). The corresponding assertion for the index of $A_{1}^{\rho, \omega}$ is well-known, see, e.g., [19]. Thus, Lemma 3.5 provides us with another proof of this result.

CoROllary 3.7. If $\omega=\pi$ and if the operator $A_{1}^{\rho, \pi}$ is Fredholm, then its index is zero.

It is obvious from Lemma 3.5 that the winding number of $\operatorname{det} \operatorname{Sym} A_{1}^{\rho, \omega}(1, \mu)$ and, thus, the index of the operator $A_{1}^{\rho, \omega}$ depend essentially on the properties of the function

$$
\Phi(\mu)=\frac{\sin ((\omega-\pi) \theta)}{\sin (\pi \theta)}, \quad \theta=\frac{1}{2}-\rho+\frac{\mathrm{i}}{2 \pi} \log \frac{\mu}{1-\mu} .
$$

Of particular interest for index computation is the location of the zeros of the real and imaginary part of the function $\Phi$ in the open interval $(0,1)$ which will be our next concern. The results of those (somewhat tedious) considerations are summarized in Lemmas 3.11 (imaginary part) and 3.17 (real part) below.

Since $\theta=\alpha+\mathrm{i} \beta$ with $\alpha=1 / 2-\rho$ and $\beta=(1 / 2 \pi) \log (\mu /(1-\mu))$, one has

$$
\begin{aligned}
\Phi(\mu)= & \frac{\sin ((\omega-\pi) \theta) \overline{\sin (\pi \theta)}}{\sin (\pi \theta) \overline{\sin (\pi \theta)}}=\frac{\sin ((\omega-\pi) \theta) \sin (\pi \bar{\theta})}{|\sin (\pi \theta)|^{2}} \\
= & \frac{\cos ((\omega-2 \pi \alpha)+\mathrm{i} \omega \beta)-\cos (\omega \alpha+\mathrm{i}(\omega-2 \pi) \beta)}{2|\sin (\pi \theta)|^{2}} \\
= & \frac{\cos ((\omega-2 \pi) \alpha) \cosh (\omega \beta)-\cos (\omega \alpha) \cosh ((\omega-2 \pi) \beta)}{2|\sin (\pi \theta)|^{2}} \\
& -\mathrm{i} \frac{\sin ((\omega-2 \pi) \beta) \sinh (\omega \beta)-\sin (\omega \alpha) \sinh ((\omega-2 \pi) \beta)}{2|\sin (\pi \theta)|^{2}} ;
\end{aligned}
$$

thus, the real and imaginary parts of the function $\Phi$ have the form

$$
\begin{aligned}
& \operatorname{Re} \Phi(\mu)=\frac{\cos ((\omega-2 \pi) \alpha) \cosh (\omega \beta)-\cos (\omega \alpha) \cosh ((\omega-2 \pi) \beta)}{2|\sin (\pi \theta)|^{2}} \\
& \operatorname{Im} \Phi(\mu)=\frac{\sin (\omega \alpha) \sinh ((\omega-2 \pi) \beta)-\sin ((\omega-2 \pi) \beta) \sinh (\omega \beta)}{2|\sin (\pi \theta)|^{2}}
\end{aligned}
$$

The common denominator of (3.10) and (3.11) can be written as

$$
\begin{aligned}
& |\sin (\pi \theta)|^{2}=\sin (\pi \theta) \sin (\pi \bar{\theta})=\frac{\cos (\pi(\theta-\bar{\theta}))-\cos (\pi(\theta+\bar{\theta}))}{2} \\
& \quad=\frac{\cosh (2 \pi \beta)-\cos (2 \pi \alpha)}{2}=\frac{1}{4}\left[\left(\frac{\mu}{1-\mu}+\frac{1-\mu}{\mu}\right)+2 \cos (2 \pi \rho)\right] \\
& \quad=\frac{(2 \mu-1)^{2}+4 \mu(1-\mu) \cos ^{2}(\pi \rho)}{4 \mu(1-\mu)}
\end{aligned}
$$


whence via straightforward calculation follows

$$
\begin{aligned}
\operatorname{Re} \Phi(\mu)= & \frac{\mu(1-\mu)}{R(\mu)}\left[\left(\left(\frac{\mu}{1-\mu}\right)^{\delta}+\left(\frac{1-\mu}{\mu}\right)^{\delta}\right) \cos (2 \pi(\delta-1) \alpha)\right. \\
& \left.-\left(\left(\frac{\mu}{1-\mu}\right)^{\delta-1}+\left(\frac{1-\mu}{\mu}\right)^{\delta-1}\right) \cos (2 \pi \delta \alpha)\right], \\
\operatorname{Im} \Phi(\mu)= & \frac{\mu(1-\mu)}{R(\mu)}\left[\left(\left(\frac{\mu}{1-\mu}\right)^{\delta-1}-\left(\frac{1-\mu}{\mu}\right)^{\delta-1}\right) \sin (2 \pi \delta \alpha)\right. \\
& \left.-\left(\left(\frac{\mu}{1-\mu}\right)^{\delta}-\left(\frac{1-\mu}{\mu}\right)^{\delta}\right) \sin (2 \pi(\delta-1) \alpha)\right]
\end{aligned}
$$

with

$$
R(\mu):=(2 \mu-1)^{2}+4 \mu(1-\mu) \cos ^{2}(\pi \rho), \quad-1 / 2<\rho<1 / 2 ;
$$

and $\delta:=\omega /(2 \pi), \quad \delta \in(0,1)$.

Let us start with considering the imaginary part of $\Phi$. For $0<\mu<1 / 2$, introduce a new variable $x$ by $x=(1-\mu) / \mu$ and set $y=2 \pi \alpha$, i.e. $x$ runs through $(1,+\infty)$, and $y$ lies in $(0,2 \pi)$. Because the function $\mu \mapsto \mu(1-\mu) / R(\mu)$ has no zeros in the interval $(0,1)$, the zeros of $\operatorname{Im} \Phi$ are completely determined by those of the function

$$
\psi_{\delta}(x):=\left(x^{1-\delta}-x^{\delta-1}\right) \sin (\delta y)+\left(x^{-\delta}-x^{\delta}\right) \sin ((1-\delta) y) .
$$

The following property of the function $\psi_{\delta}$ is obvious:

Lemma 3.8. Let $\delta$ be in $(1 / 2,1)$ and $y=\pi / \delta$. Then the function $\psi_{\delta}$ has no zeros in $(1, \infty)$.

Thus, when investigating the function $\psi_{\delta}$ in case $\delta>1 / 2$, we can restrict ourselves to the case when $y \neq \pi / \delta$.

Write the function $\psi_{\delta}$ as

$$
\psi_{\delta}(x)=\sin (\delta y)\left(x^{-\delta}-x^{\delta}\right)\left(\phi_{\delta}(x)-A_{\delta}(y)\right)
$$

with

$$
A_{\delta}(y):=\frac{\sin ((1-\delta) y)}{\sin (\delta y)} \text { and } \phi_{\delta}(x):=\frac{x^{1-\delta}-x^{\delta-1}}{x^{-\delta}-x^{\delta}}
$$

Further, given a function $f$, denote by $I_{f}(a, b)$ the image of the interval $(a, b)$ under that function.

Lemma 3.9. Let $\phi_{\delta}$ be the function defined in (3.16). Then

$$
I_{\phi_{\delta}}(1, \infty)=\left\{\begin{array}{lll}
((1-\delta) / \delta, \infty) & \text { if } & \delta \in(0,1 / 2) \\
(0,(1-\delta) / \delta) & \text { if } & \delta \in(1 / 2,1)
\end{array} .\right.
$$

Proof. The function $\phi_{\delta}$ is continuous on $(1, \infty)$ with one-sided limits

$$
\lim _{x \rightarrow 1+0} \phi_{\delta}(x)=(1-\delta) / \delta, \quad \lim _{x \rightarrow+\infty} \phi_{\delta}(x)=\left\{\begin{array}{lll}
+\infty & \text { if } & \delta \in(0,1 / 2) \\
0 & \text { if } & \delta \in(1 / 2,1)
\end{array},\right.
$$


and the elementary inequality

$$
\frac{x^{\alpha}-x^{-\alpha}}{\alpha}<\frac{x^{\beta}-x^{-\beta}}{\beta},
$$

which is valid for $x>1$ and $0<\alpha<\beta<1$, yields

$$
\phi_{\delta}(x)<\frac{1-\delta}{\delta} \quad \text { if } x \in(1, \infty) \text { and } \delta \in(0,1 / 2),
$$

and

$$
\frac{1-\delta}{\delta}<\phi_{\delta}(x) \quad \text { if } x \in(1, \infty) \text { and } \delta \in(1 / 2,1)
$$

(put $\alpha=\delta, \beta=1-\delta$ and $\alpha=1-\delta, \beta=\delta$ in (3.18), respectively).

A similar discussion of the function $A_{\delta}$ gives

Lemma 3.10. Let $A_{\delta}$ be defined by (3.16) and let $y \in(0,2 \pi)$.

(a) If $\delta \in(0,1 / 2)$, then $A_{\delta}(y)<(1-\delta) / \delta$ for all $y \in(0,2 \pi)$.

(b) If $\delta \in(1 / 2,1)$, then $A_{\delta}(y)>(1-\delta) / \delta$ for all $y \in(0, \pi / \delta)$, and $A_{\delta}(y)<0$ for all $y \in(\pi / \delta, 2 \pi)$.

Proof. For $0<\alpha<\beta<\pi$ one has

$$
\frac{\sin \beta}{\beta}<\frac{\sin \alpha}{\alpha}
$$

If $\delta \in(0,1 / 2)$, and if we set $\alpha=\delta y$ and $\beta=(1-\delta) y$ in (3.19), then this inequality yields for all $y \in(0, \pi /(1-\delta))$

$$
A_{\delta}(y)=\frac{\sin ((1-\delta) y)}{\sin (\delta y)}<\frac{1-\delta}{\delta}
$$

which shows that assertion (a) is correct in this case, whereas in case $y \in(\pi /(1-$ $\delta), 2 \pi)$ one has $\sin ((1-\delta) y) \leq 0$ which verifies (a) for those $y$, too.

If we analogously put $\alpha=(1-\delta) y$ and $\beta=\delta y$ with $y \in(0, \pi / \delta)$ in (3.19), then we get the first estimate in (b), and the second one is evident.

Lemma 3.11. Let $\delta \in(0,1 / 2) \cup(1 / 2,1)$, and $\rho \in(-1 / 2,1 / 2)$. Then the function $\mu \mapsto \operatorname{Im} \Phi(\mu)$ has no zero in the intervals $(0,1 / 2)$ and $(1 / 2,1)$.

Proof. The assertion concerning the interval $(0,1 / 2)$ follows from Lemmas 3.8, 3.9 and 3.10 , and for $(1 / 2,1)$ one can make use of the evident identity

$$
\operatorname{Im} \Phi(\mu)=-\operatorname{Im} \Phi(1-\mu), \quad \mu \in(0,1 / 2) .
$$

Now we turn over to the real part of the function $\Phi$ which is given by (3.12). As above, we introduce a new variable $x:=(1-\mu) / \mu$, set $y:=2 \pi \alpha$, and consider the function

$$
\hat{\psi}_{\delta}(x)=\left(x^{\delta}+x^{-\delta}\right) \cos ((\delta-1) y)-\left(x^{\delta-1}+x^{1-\delta}\right) \cos (\delta y), \quad x \in(1,+\infty) .
$$

For $\delta \in(1 / 4,1 / 2) \cup(1 / 2,3 / 4)$, there is only one number $y \in(0,2 \pi)$ such that $\cos (\delta y)$ vanishes, whereas in case $\delta \in(3 / 4,1)$ there are exactly two different zeros $y_{1}, y_{2} \in$ 
$(0,2 \pi)$ of the equation $\cos (\delta y)=0$. But in any case, if $\cos (\delta y)=0$, then the sign of the function $\hat{\psi}_{\delta}$ is constant on the interval $(1, \infty)$. Therefore, in what follows we suppose that $y \in(0,2 \pi)$ as well as $\delta \in(0,1 / 2) \cup(1 / 2,1)$ are chosen in such a way that $\cos (\delta y) \neq 0$. Under this assumption, the function $\hat{\psi}_{\delta}$ can be written as

$$
\hat{\psi}_{\delta}(x)=\left(x^{\delta}+x^{-\delta}\right) \cos (\delta y)\left(\hat{A}_{\delta}-\hat{\phi}_{\delta}(x)\right)
$$

where

$$
\hat{\phi}_{\delta}(x)=\frac{x^{1-\delta}+x^{\delta-1}}{x^{\delta}+x^{-\delta}} \quad \text { and } \quad \hat{A}_{\delta}=\hat{A}_{\delta}(y)=\frac{\cos ((\delta-1) y)}{\cos (\delta y)}
$$

LEMMA 3.12. Let $\hat{\phi}_{\delta}(x)$ be the function defined in (3.21). Then

$$
I_{\hat{\phi}_{\delta}}(1, \infty)=\left\{\begin{array}{lll}
(1, \infty) & \text { if } & \delta \in(0,1 / 2) \\
(0,1) & \text { if } & \delta \in(1 / 2,1) .
\end{array}\right.
$$

The proof is the same as that of Lemma 3.9 with the only difference that the inequality

$$
x^{\beta}+x^{-\beta}<x^{\alpha}+x^{-\alpha} \text { for all } 0<\beta<\alpha<1 \text { and } x>1
$$

should be used instead of (3.18).

Let us now examine the function $\hat{A}_{\delta}$ given by (3.21). The behaviour of the derivative

$$
\hat{A}_{\delta}^{\prime}(y)=\frac{\sin ((2 \delta-1) y)+(2 \delta-1) \sin y}{2 \cos ^{2}(\delta y)}
$$

of that function depends essentially on the parameter $\delta$. Therefore, the cases $\delta \in$ $(0,1 / 4], \delta \in(1 / 4,1 / 2), \delta \in(1 / 2,3 / 4)$ and $\delta \in[3 / 4,1)$ will be considered separately in the subsequent four lemmata.

Lemma 3.13. If $\delta \in(0,1 / 4]$, then $I_{\hat{A}_{\delta}}(0,2 \pi) \cap I_{\hat{\phi}_{\delta}}(1, \infty)=\emptyset$.

Proof. To start with, suppose $\delta \in(0,1 / 4)$. Let $\hat{\eta}_{\delta}$ denote the numerator of (3.22). Its derivative

$$
\hat{\eta}_{\delta}^{\prime}(y)=2(2 \delta-1) \cos (\delta y) \cos ((1-\delta) y)
$$

has the two zeros

$$
y_{1}:=\frac{\pi}{2(1-\delta)} \in\left(\frac{\pi}{2}, \frac{2 \pi}{3}\right), \quad y_{2}:=\frac{3 \pi}{2(1-\delta)} \in\left(\frac{3 \pi}{2}, 2 \pi\right),
$$

in $(0,2 \pi)$, and $\hat{\eta}_{\delta}^{\prime}(y)$ is negative for $y \in\left(0, y_{1}\right) \cup\left(y_{2}, 2 \pi\right)$ and positive for $y \in\left(y_{1}, y_{2}\right)$. Particularly, $\hat{\eta}_{\delta}$ is a monotonically decreasing function on both intervals $\left(0, y_{1}\right)$ and $\left(y_{2}, 2 \pi\right)$. Since $\hat{\eta}_{\delta}(+0)=0$ and $\hat{\eta}_{\delta}(2 \pi-0)=\sin (4 \pi \delta)>0$, one further has

$$
\hat{A}_{\delta}^{\prime}(y)<0 \text { for all } y \in\left(0, y_{1}\right) \text { and } \hat{A}_{\delta}^{\prime}(y)>0 \text { for all } y \in\left(y_{2}, 2 \pi\right) \text {. }
$$

These inequalities imply that the function $\hat{A}_{\delta}$ is monotonically decreasing on $\left(0, y_{1}\right)$ and monotonically increasing on $\left(y_{2}, 2 \pi\right)$. Since $\hat{A}_{\delta}(+0)=\hat{A}_{\delta}(2 \pi-0)=1$ we thus 
obtain $\hat{A}_{\delta}(y)<1$ for all $y \in\left(0, y_{1}\right) \cup\left(y_{2}, 2 \pi\right)$. Moreover, it is easy to see that $\hat{A}_{\delta}(y) \leq 0$ for all $y \in\left[y_{1}, y_{2}\right]$. Hence, $I_{\hat{A}_{\delta}}(0,2 \pi) \subseteq(-\infty, 1)$, which proves together with Lemma 3.12 the claim in case $\delta \in(0,1 / 4)$. If, finally, $\delta=1 / 4$, then $y_{2}=2 \pi$, and $\hat{A}_{\delta}(y)$ is negative everywhere on the interval $\left(y_{1}, 2 \pi\right)$.

LEMMA 3.14. Let $\delta \in(1 / 4,1 / 2)$ and set $y_{1}:=\pi /(2 \delta) \in(\pi, 2 \pi)$ and $y_{2}:=$ $\pi /(2(1-\delta)) \in(2 \pi / 3, \pi)$. Then

(a) $I_{\hat{A}_{\delta}}\left(0, y_{1}\right) \cap I_{\hat{\phi}_{\delta}}(1, \infty)=\emptyset$.

(b) $I_{\hat{A}_{\delta}}\left(y_{1}, 2 \pi\right) \subseteq I_{\hat{\phi}_{\delta}}(1, \infty)$. Moreover, for every $y \in\left(y_{1}, 2 \pi\right)$, there is only one $x \in(1, \infty)$ such that $\hat{\phi}_{\delta}(x)=\hat{A}_{\delta}(y)$.

Proof. The points $y_{1}$ and $y_{2}$ are zeros of the derivative $\hat{\eta}_{\delta}^{\prime}$ in (3.23), and $\hat{\eta}_{\delta}^{\prime}(y)<0$ for all $y \in\left(0, y_{2}\right) \cup\left(y_{1}, 2 \pi\right)$ but $\hat{\eta}_{\delta}^{\prime}(y)>0$ if $y \in\left(y_{2}, y_{1}\right)$. Hence, the function $\hat{\eta}_{\delta}$ is monotonically decreasing on $\left(0, y_{2}\right) \cup\left(y_{1}, 2 \pi\right)$ and monotonically increasing on $\left(y_{2}, y_{1}\right)$. Since $\hat{\eta}_{\delta}(+0)=0$ and $\hat{\eta}_{\delta}\left(y_{1}\right)=2 \delta \sin (\pi / 2 \delta)<0$, the derivative $\hat{A}_{\delta}^{\prime}$ is negative on $\left(0, y_{1}\right) \cup\left(y_{1}, 2 \pi\right)$. Therefore, the function $\hat{A}_{\delta}$ is monotonically decreasing on the intervals $\left(0, y_{1}\right)$ and $\left(y_{1}, 2 \pi\right)$. Moreover, $\hat{A}_{\delta}(+0)=1$. Combining these observations with Lemma 3.12, we obtain assertion (a).

Now consider the function $\hat{A}_{\delta}$ on the interval $\left(y_{1}, 2 \pi\right)$. It is continuous there, and has the one-sided limits $\hat{A}_{\delta}\left(y_{1}+0\right)=\infty$ and $\hat{A}_{\delta}(2 \pi-0)=1$. Taking into account Lemma 3.12 once again, we find that $\hat{A}_{\delta}(y) \in I_{\hat{\phi}_{\delta}}(1, \infty)$ for every $y \in\left(y_{1}, 2 \pi\right)$, and the uniqueness of an element $x$ such that $\hat{\phi}_{\delta}(x)=\hat{A}_{\delta}(y)$ follows from the strict monotonicity of the function $\hat{\phi}_{\delta}$.

Analogous discussions lead to the following two lemmas.

LEMMA 3.15. Let $\delta \in(1 / 2,3 / 4)$, and set $y_{1}:=\pi /(2 \delta) \in(2 \pi / 3, \pi)$ and $y_{2}:=$ $\pi /(2(1-\delta)) \in(\pi, 2 \pi)$. Then

(a) $I_{\hat{A}_{\delta}}\left(\left(0, y_{1}\right) \cup\left(y_{1}, y_{2}\right)\right) \cap I_{\hat{\phi}_{\delta}}(1, \infty)=\emptyset$.

(b) $I_{\hat{A}_{\delta}}\left(y_{2}, 2 \pi\right) \subseteq I_{\hat{\phi}_{\delta}}(1, \infty)$. Moreover, for every $y \in\left(y_{2}, 2 \pi\right)$, there is only one $x \in(1, \infty)$ such that $\hat{\phi}_{\delta}(x)=\hat{A}_{\delta}(y)$.

LEMMA 3.16. Let $\delta \in(3 / 4,1)$ and set $y_{1}:=\pi /(2 \delta)$ and $y_{2}:=3 \pi /(2 \delta)$. Then

$$
I_{\hat{A}_{\delta}}\left(\left(0, y_{1}\right) \cup\left(y_{1}, y_{2}\right) \cup\left(y_{2}, 2 \pi\right)\right) \cap I_{\hat{\phi}_{\delta}}(1, \infty)=\emptyset \text {. }
$$

Summarizing the preceding lemmas and taking into account the identity

$$
\operatorname{Re} \Phi(1-\mu)=\operatorname{Re} \Phi(\mu), \quad \text { for } \mu \in(0,1 / 2)
$$

we arrive at the following characterization of the zeros of the real part of the function $\Phi$.

LEMMA 3.17. Let $\Phi$ be the function defined in Lemma 3.5.

(a) If $\delta \in\left(0, \frac{1}{4}\right] \cup\left[\frac{3}{4}, 1\right)$ and $\rho \in\left(-\frac{1}{2}, \frac{1}{2}\right)$, then the real part $\operatorname{Re} \Phi$ of the function $\Phi$ has no zeros in $\left(0, \frac{1}{2}\right) \cup\left(\frac{1}{2}, 1\right)$.

(b) If $\delta \in\left(\frac{1}{4}, \frac{1}{2}\right)$ and $\rho \in\left[\frac{1}{2}-\frac{1}{4 \delta}, \frac{1}{2}\right)$, then $\operatorname{Re} \Phi$ has no zeros on $\left(0, \frac{1}{2}\right) \cup\left(\frac{1}{2}, 1\right)$, 
whereas in case $\rho \in\left(-\frac{1}{2}, \frac{1}{2}-\frac{1}{4 \delta}\right)$ the function $\operatorname{Re} \Phi$ has exactly one zero in $\left(0, \frac{1}{2}\right)$ and one in $\left(\frac{1}{2}, 1\right)$.

(c) If $\delta \in\left(\frac{1}{2}, \frac{3}{4}\right)$ and $\rho \in\left[\frac{1}{2}-\frac{1}{4(1-\delta)}\right.$, $\left.\frac{1}{2}\right)$, then $\operatorname{Re} \Phi$ has no zeros on $\left(0, \frac{1}{2}\right) \cup\left(\frac{1}{2}, 1\right)$, whereas in case $\rho \in\left(-\frac{1}{2}, \frac{1}{2}-\frac{1}{4(1-\delta)}\right)$, the function $\operatorname{Re} \Phi$ has exactly one zero in $\left(0, \frac{1}{2}\right)$ and one in $\left(\frac{1}{2}, 1\right)$.

Corollary 3.18. If $\delta \in(0,1 / 2) \cup(1 / 2,1)$ and $\rho \geq 0$, then the function $\operatorname{Re} \Phi$ has no zeros on $(0,1 / 2) \cup(1 / 2,1)$.

Here is the main result of this section.

THEOREM 3.19. Let $\rho \in(-1 / 2,1 / 2), \omega \in(0,2 \pi)$, and let the operator $A_{1}^{\rho, \omega}$ be Fredholm. Then the index $\kappa:=\kappa\left(A_{1}^{\rho, \pi}\right)$ of $A_{1}^{\rho, \omega}$ satisfies the estimates summarized in the following table. This table has to be read as follows: If the angle $\omega$ and the weight $\rho$ belong to the mentioned intervals, then $\kappa$ is subject to the corresponding estimate in the last column.

\begin{tabular}{|l|l|l|}
\hline Angle & Weight & Index \\
\hline $0<\omega \leq \frac{\pi}{2}$ & $-\frac{1}{2}<\rho<\frac{1}{2}$ & $|\kappa| \leq 1$ \\
$\frac{\pi}{2}<\omega<\pi$ & $-\frac{1}{2}<\rho<\frac{1}{2}-\frac{\pi}{2 \omega}$ & $|\kappa| \leq 2$ \\
$\frac{\pi}{2}<\omega<\pi$ & $\frac{1}{2}-\frac{\pi}{2 \omega} \leq \rho<\frac{1}{2}$ & $|\kappa| \leq 1$ \\
$\omega=\pi$ & $-\frac{1}{2}<\rho<\frac{1}{2}$ & $|\kappa|=0$ \\
$\pi<\omega<\frac{3 \pi}{2}$ & $-\frac{1}{2}<\rho<\frac{1}{2}-\frac{\pi}{2(2 \pi-\omega)}$ & $|\kappa| \leq 2$ \\
$\pi<\omega<\frac{3 \pi}{2}$ & $\frac{1}{2}-\frac{\pi}{2(2 \pi-\omega)} \leq \rho<\frac{1}{2}$ & $|\kappa| \leq 1$ \\
$\frac{3 \pi}{2} \leq \omega<2 \pi$ & $-\frac{1}{2}<\rho<\frac{1}{2}$ & $|\kappa| \leq 1$ \\
\hline
\end{tabular}

Proof. Lemma 3.5 implies that the imaginary part of the determinant of the symbol of $A_{1}^{\rho, \omega}$ has the form

$$
\operatorname{Im} \operatorname{det}\left\{\operatorname{Sym} A_{1}^{\rho, \omega}(1, \mu)\right\}=-8|N|^{2} \operatorname{Im} \Phi(\mu) \operatorname{Re} \Phi(\mu) .
$$

According to Lemmas 3.11 and 3.17 , the function $\operatorname{Im} \Phi \cdot \operatorname{Re} \Phi$ can possess either three $(\mu=0, \mu=1 / 2, \mu=1)$ or five zeros on $[0,1]$. Since $\operatorname{det}\left\{\operatorname{Sym} A_{1}^{\rho, \omega}(t, \mu)\right\}$ is real for $t \neq 1$ and $\mu \in[0,1]$ (cf. Lemma 3.3), the curve

$$
\left\{\operatorname{det}\left\{\operatorname{Sym} A_{1}^{\rho, \omega}(1, \mu)\right\}, 0 \leq \mu \leq 1\right\} \cup\left\{\operatorname{det}\left\{\operatorname{Sym} A_{1}^{\rho, \omega}(t, 0)\right\}, t \neq 1\right\}
$$

can perform no more than one (in case of three zeros) or two (in case of five zeros) rotations arround the origin.

Corollary 3.20. Let $\omega \in(0,2 \pi), \rho \in[0,1 / 2)$, and let the operator $A_{1}^{\rho, \omega}$ be Fredholm. Then $\left|\kappa\left(A_{1}^{\rho, \omega}\right)\right| \leq 1$.

Figure 1 shows the image of the interval $[0,1]$ under the function $\Phi^{2}$ in the case $\omega=5 \pi / 4, \rho=-0.49$. Observe that at the first glance, this figure does not give any hint that there might be a second coil of the curve. However, after Theorem 3.19 was proved we returned to this example and, as the result of a more careful consideration, we observed that, in a neighbourhood of the origin, the curve actually behaves as in Figure 2.

4. On the vanishing of the index of the local operators. Here we are going to discuss criteria for the vanishing of the index $\kappa\left(A_{1}^{\rho, \omega}\right)$, which is a necessary condition for the applicability of the modified quadrature methods considered in Section 5 . The following result shows indeed that, in case the estimate $\left|\kappa\left(A_{1}^{\rho, \omega}\right)\right| \leq 1$ can be 


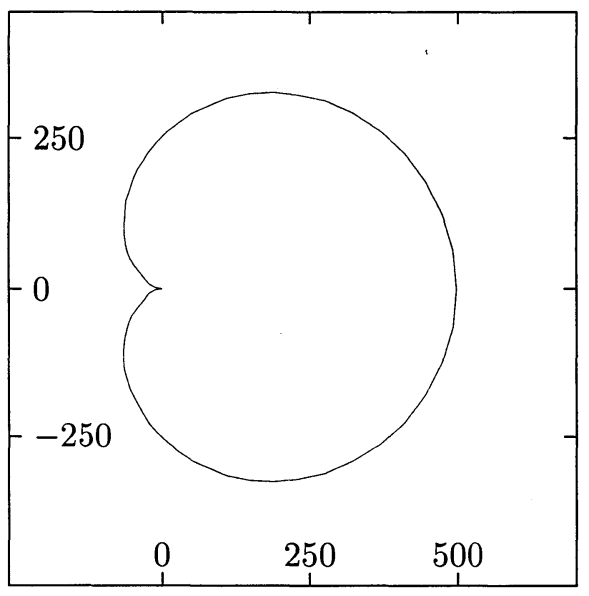

Figure 1

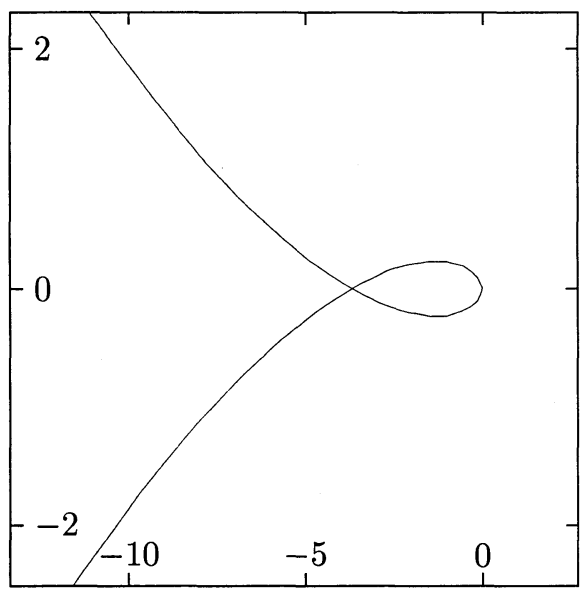

Figure 2

guaranteed, it is possible to force that $\kappa\left(A_{1}^{\rho, \omega}\right)$ vanishes by appropriately choosing the weight function $\rho$. Recall that $U$ and $V$ are given by (3.9).

TheOREM 4.1. Let $\omega \in(0, \pi) \cup(\pi, 2 \pi)$, assume the operator $A_{1}^{0, \omega}$ to be Fredholm, and let one of the following conditions be satisfied:

(i) $\quad \omega \in(0, \pi] \cup[3 \pi / 2,2 \pi)$ and $\rho \in(-1 / 2,1 / 2)$,

(ii) $\quad \omega \in(\pi / 2, \pi)$ and $\rho \in[-1 / 2-\pi /(2 \omega), 1 / 2)$, or

(iii) $\omega \in(\pi, 3 / 2 \pi)$ and $\rho \in[-1 / 2-\pi /(2(2 \pi-\omega)), 1 / 2)$.

Then the following assertions are true:

(a) The operator $A_{1}^{\rho, \omega}$ is Fredholm if and only if

$$
t_{2}=t_{2}(\rho):=t_{1}-4|c b-a d|^{2} \frac{\sin ^{2}((\omega-\pi)(1 / 2-\rho))}{\cos ^{2}(\pi \rho)} \neq 0
$$

with $t_{1}:=U=\left|a^{2}-b^{2}\right|^{2}+\left|c^{2}-d^{2}\right|^{2}+2\left(|c b-a d|^{2}-|a c-b d|^{2}\right)$.

(b) If the operator $A_{1}^{\rho, \omega}$ is Fredholm, then its index vanishes if and only if $t_{1} \cdot t_{2}>0$.

Proof. Let $A_{1}^{0, \omega}$ be a Fredholm operator. Then $t \mapsto \operatorname{det}\left\{\operatorname{Sym} A_{1}^{0, \omega}(t, 0)\right\}, t \neq 1$, is a real-valued function without zero. Since

$$
\left.\operatorname{det}\left\{\operatorname{Sym} A_{1}^{\rho, \omega}(t, 0)\right\}\right|_{t \neq 1}=\left.\operatorname{det}\left\{\operatorname{Sym} A_{1}^{0, \omega}(t, 0)\right\}\right|_{t \neq 1},
$$

the operator $A_{1}^{\rho, \omega}$ is Fredholm if and only if the origin does not lie on the arc

$$
\tilde{\Gamma}:=\left\{\operatorname{det}\left\{\operatorname{Sym} A_{1}^{\rho, \omega}(1, \mu)\right\}, \mu \in[0,1]\right\}=\left\{U+V \Phi^{2}(\mu), \mu \in[0,1]\right\} .
$$

Each of the conditions (i) - (iii) implies that $\tilde{\Gamma}$ possesses only two points in common with the real axis which correspond to the parameters $\mu=0$ and $\mu=1 / 2$. We denote these points by $\tilde{t}_{1}$ and $\tilde{t}_{2}$. Since

$$
\Phi^{2}(\mu)=(\operatorname{Re} \Phi(\mu))^{2}-(\operatorname{Im} \Phi(\mu))^{2}+2 \operatorname{Re} \Phi(\mu) \operatorname{Im} \Phi(\mu)
$$

with $\operatorname{Re} \Phi$ and $\operatorname{Im} \Phi$ given by (3.12) and (3.13), respectively, and since

$$
\operatorname{Re} \Phi(0)=\operatorname{Im} \Phi(0)=\operatorname{Im} \Phi(1 / 2)=0,
$$




$$
\operatorname{Re} \Phi(1 / 2)=\frac{\sin ((\omega-\pi)(1 / 2-\rho))}{\cos (\pi \rho)} .
$$

we conclude that $\tilde{t}_{1}=t_{1}$ and $\tilde{t}_{2}=t_{2}$. However, $t_{1}$ cannot be zero since $A_{1}^{0, \omega}$ is a Fredholm operator. This finishes the proof of assertion (a), and that of (b) is obvious now.

Thus, under each of the hypotheses (i) - (iii) of Theorem 4.1, the operator $A_{1}^{\rho, \omega}$ is Fredholm, and if $t_{1} \cdot t_{2}<0$, then necessarily $\kappa\left(A_{1}^{\rho, \omega}\right) \neq 0$. Obviously, since the location of the point $t_{2}$ on the real line depends on the weight $\rho$, one might try in this case to look for some new weight $\tilde{\rho}$ such that $\kappa\left(A_{1}^{\tilde{\rho}, \omega}\right)=0$. The following corollary establishes conditions when such a choice is possible.

Corollary 4.2. Let one of conditions (i) - (iii) of the previous theorem be satisfied, and suppose that $t_{1} \cdot t_{2}<0$. Then one can choose a weight $\rho \in(-1 / 2,1 / 2)$ such that $\kappa\left(A_{1}^{\rho, \omega}\right)=0$ if and only if one of the following conditions is fulfilled:

(a) $\omega \in(0, \pi / 2] \cup[3 \pi / 2,2 \pi)$ and

$$
0 \in\left(-\infty, U-4|c b-a d|^{2}(1-\pi / \omega)^{2}\right) .
$$

(b) $\omega \in(\pi / 2, \pi)$ and

$$
0 \in\left(U-4|c b-a d|^{2} \cot ^{2}\left(\pi^{2} / 2 \omega\right), U-4|c b-a d|^{2}(1-\pi / \omega)^{2}\right) .
$$

(c) $\omega \in(\pi, 3 \pi / 2)$ and

$$
0 \in\left(U-4|c b-a d|^{2} \cot ^{2}\left(\pi^{2} / 2(2 \pi-\omega)\right), U-4|c b-a d|^{2}(1-\pi / \omega)^{2}\right) .
$$

In particular, if $U>0$ but $U-4|c b-a d|^{2}(1-\pi / \omega)^{2}<0$, then for every $\rho \in$ $(-1 / 2,1 / 2)$ which satisfies the conditions of Theorem 4.1 , the index of $\kappa\left(A_{1}^{\rho, \omega}\right)$ is not equal to zero. Conversely, if $U>0$ and $U-4|c b-a d|^{2}(1-\pi / \omega)^{2}>0$, then there always exists a $\rho$ such that all conditions of Theorem 4.1 are satisfied and that $\kappa\left(A_{1}^{\rho, \omega}\right)=0$.

Proof of Corollary 4.2. To prove the claim requires describing the range of the function

$$
\rho \mapsto(\operatorname{Re} \Phi(1 / 2)(\rho))^{2}=\left(\frac{\sin ((\omega-\pi)(1 / 2-\rho))}{\cos (\pi \rho)}\right)^{2}
$$

or equivalently, after substituting $y:=\pi(1 / 2-\rho) \in(0, \pi)$ and $\delta:=\omega / 2 \pi$, that of the function

$$
\psi_{\delta}^{2}(y):=\left(\frac{\left.\sin ((2 \delta-1) y)^{2}\right)}{\sin y}\right)^{2}
$$

The one-sided limits of $\psi_{\delta}$ at 0 and $\pi$ are

$$
\lim _{y \rightarrow+0} \psi_{\delta}(y)=2 \delta-1 \text { and } \lim _{y \rightarrow \pi-0} \psi_{\delta}(y)=\left\{\begin{array}{ccc}
-\infty & \text { if } & 2 \delta-1<0 \\
+\infty & \text { if } & 2 \delta-1>0
\end{array}\right.
$$

respectively, the derivative of $\psi_{\delta}(y)$ is

$$
\psi_{\delta}^{\prime}(y)=\frac{(\delta-1) \sin (2 \delta y)+2 \delta(\sin (1-\delta) y)}{\sin ^{2} y},
$$


and the derivative of the nominator $\eta_{\delta}(y)$ of that fraction is given by

$$
\eta_{\delta}^{\prime}(y)=4 \delta(1-\delta) \sin y \sin ((2 \delta-1) y)
$$

The following considerations depend on the location of $\delta$ in $(0,1)$.

Let $\delta \in(0,1 / 4)$. Then $\eta_{\delta}^{\prime}(y)<0$ for all $y \in(0, \pi)$, hence, $\eta_{\delta}$ is monotonically decreasing on $[0, \pi]$. Since $\eta_{\delta}(0)=0$, the function $\eta_{\delta}$ is negative on $(0, \pi)$, which implies that $\psi_{\delta}^{\prime}(y)<0$ for all $y \in(0, \pi)$. Thus, $\psi_{\delta}$ is monotonically decreasing on this interval, which together with (4.2) shows that $I_{\psi_{\delta}}(0, \pi)=(-\infty, 2 \delta-1)$ and

$$
I_{\psi_{\delta}}(0, \pi)=\left((2 \delta-1)^{2}, \infty\right) \text { if } \delta \in(0,1 / 4)
$$

If $\delta=1 / 4$, then $\psi_{1 / 4}(y)=-1 /(2 \cos (y / 2))$, which immediately gives

$$
I_{\psi_{1 / 4}^{2}}(0, \pi)=(1 / 4, \infty)
$$

In case $\delta \in(1 / 4,1 / 2)$ we have $\eta_{\delta}^{\prime}(y)<0$ for all $y \in(0, \pi)$ again. However, now we have to restrict ourselves to those $y$ for which the curve $\Gamma_{0}$ has no more than two points in common with the real line (since otherwise, we have no information about the location of the zeros of the symbol). Thus, according to Theorem 4.1, we get $y \in(0, \pi /(4 \delta))$, and therefore $I_{\psi_{\delta}}(0, \pi /(4 \delta))$ is the interval $\left(\cot \frac{\pi}{4 \delta}, 2 \delta-1\right)$, whence follows

$$
I_{\psi_{\delta}^{2}}(0, \pi /(4 \delta))=\left((2 \delta-1)^{2}, \cot ^{2} \frac{\pi}{4 \delta}\right) \quad \text { if } \delta \in(1 / 4,1 / 2) .
$$

Analogously we obtain

$$
\begin{gathered}
I_{\psi_{\delta}^{2}}(0, \pi /(4(1-\delta)))=\left((2 \delta-1)^{2}, \cot ^{2} \frac{\pi}{4(1-\delta)}\right) \quad \text { if } \delta \in(1 / 2,3 / 4) \\
I_{\psi_{3 / 4}^{2}}(0, \pi)=(1 / 4, \infty) \\
I_{\psi_{\delta}^{2}}(0, \pi)=\left((2 \delta-1)^{2}, \infty\right) \text { if } \delta \in(3 / 4,1)
\end{gathered}
$$

A comparison of (4.3) - (4.8) with (4.1) indicates that the conditions of Corollary 4.2 indeed allow to choose the weight parameter $\rho$ in such a way that the product $t_{1} \cdot t_{2}(\rho)$ becomes positive and, hence the index of $A_{1}^{\rho, \omega}$ becomes zero..

5. Modification of the quadrature method by cutting off corner singularities. In case the operator $A$ is invertible, and the local operators $A_{1, \tau}$ are Fredholm operators with index 0 but not invertible, then the quadrature rule (2.2) can be modified in such a way that the necessary and sufficient stability conditions become essentially weaker than for the original system. The modification under consideration was proposed by Chandler and Graham [12] and consists in cutting off the quadrature rule in the neighbourhood of the corner points. For, choose a non-negative integer $i_{0}$, define a set of subscripts $I_{n, i_{0}}$ as the set of all $k$ in $\{0,1, \ldots, n-1\}$ such that

$$
\left|k / n-j / n_{0}\right| \geq i_{0} / n \text { for every } j=1,2, \ldots, n_{0}-1 \text {, }
$$


and replace (2.2) by the system

$$
\begin{aligned}
& {\left[a\left(\tau_{k}^{(n)}\right)-b\left(\tau_{k}^{(n)}\right) i \cot (\pi(\varepsilon-\delta))\right] \xi_{k}^{(n)}+\frac{b\left(\tau_{k}^{(n)}\right)}{\pi i} \sum_{j \in I_{n, i_{0}}} \frac{\Delta t_{j}^{(n)}}{t_{j}^{(n)}-\tau_{k}^{(n)}} \xi_{j}^{(n)}} \\
& +\left[c\left(\tau_{k}^{(n)}\right)-d\left(\tau_{k}^{(n)}\right) i \cot (\pi(\varepsilon-\delta))\right] \overline{\xi_{k}^{(n)}}+\frac{d\left(\tau_{k}^{(n)}\right)}{\pi i} \sum_{j \in I_{n, i_{0}}} \frac{\Delta t_{j}^{(n)}}{t_{j}^{(n)}-\tau_{k}^{(n)}} \overline{\xi_{j}^{(n)}} \\
& +\sum_{j \in I_{n, i_{0}}} k_{1}\left(t_{k}^{(n)}, t_{j}^{(n)}\right) \Delta t_{j}^{(n)} \xi_{j}^{(n)}+\sum_{j \in I_{n, i_{0}}} k_{2}\left(t_{k}^{(n)}, t_{j}^{(n)}\right) \Delta t_{j}^{(n)} \overline{\xi_{j}^{(n)}} \\
& =f\left(\tau_{k}^{(n)}\right), \quad k \in I_{n, i_{0}} .
\end{aligned}
$$

Observe that the choice $i_{0}=0$ yields the original system (2.2).

The modified method can be analysed in a similar way as the original one (compare Sections 4.2.4-4.2.6 in [14] for details), and what results is the following theorem where $r_{\tau}=(\alpha-1) / 2-\rho_{\tau} \alpha$ again, where $P_{i}$ stands for the projection operator

$$
P_{i}: l_{r_{\tau}}^{2} \rightarrow l_{r_{\tau}}^{2}, \quad\left(x_{0}, x_{1}, \ldots\right) \mapsto\left(x_{0}, \ldots, x_{i-1}, 0,0, \ldots\right)
$$

in case $i \geq 1$, and where $Q_{i}:=I-P_{i}$. The same notations $P_{i}$ and $Q_{i}$ will be used for the diagonal operators $\operatorname{diag}\left(P_{i}, P_{i}, P_{i}, P_{i}\right)$ and $\operatorname{diag}\left(Q_{i}, Q_{i}, Q_{i}, Q_{i}\right)$, respectively.

THEOREM 5.1. The modified quadrature method (5.1) is stable if and only if

(a) the operator $A$ is invertible,

(b) the local operators $A_{1, \tau}$ are invertible for all $\tau \in \Gamma \backslash\left\{\gamma\left(u_{1}\right), \ldots, \gamma\left(u_{n_{0}}\right)\right\}$, and

(c) the sequences $\left(P_{i}+Q_{i} A_{1, \tau} Q_{i}\right)_{i \geq 1}$ are stable for all $\tau \in\left\{\gamma\left(u_{1}\right), \ldots, \gamma\left(u_{n_{0}}\right)\right\}$.

Observe that, if the sequence $\left(P_{i}+Q_{i} A_{1, \tau} Q_{i}\right)_{i \geq 1}$ is stable, then the operators $P_{i}+$ $Q_{i} A_{1, \tau} Q_{i}$ are invertible for sufficiently large $i$, hence, $A_{1, \tau}$ is necessarily a Fredholm operator with index 0 in that case (due to the compactness of the $P_{i}$ ). On the other hand, the invertibility of $A_{1, \tau}$ is not necessary for the stability of that sequence since the strong limit of the operators $P_{i}+Q_{i} A_{1, \tau} Q_{i}$ as $i \rightarrow \infty$ is the identity operator (which evidently is invertible). In that sense, $\left(P_{i}+Q_{i} A_{1, \tau} Q_{i}\right)_{i \geq 1}$ is an approximation method for the identity operator.

The operator $A_{1, \tau}$ is of the form Toeplitz operator + discretized Mellin convolution; thus Theorem 4.4 in [14] applies to give the following stability result for the sequence $\left(P_{i}+Q_{i} A_{1, \tau} Q_{i}\right)_{i \geq 1}$ (compare also Example 4.7 in [14]). In this theorem, we again make use of the abbreviations $a:=a(\tau), b:=b(\tau), c:=c(\tau), d:=d(\tau)$ introduced in Section 3.

THEOREM 5.2. The sequence $\left(P_{i}+Q_{i} A_{1, \tau} Q_{i}\right)_{i \geq 1}$ is stable if and only if the following conditions are satisfied:

(a) the operator $A_{1, \tau}$ is Fredholm on $l_{r_{\tau}}^{2} \oplus \ldots \oplus l_{r_{\tau}}^{2}$,

(b) the $4 \times 4$ block Toeplitz operator $T$ with generating function

$$
\left(\begin{array}{cccc}
a+b f^{(\varepsilon-\delta)} & 0 & c+d f^{(\varepsilon-\delta)} & 0 \\
0 & a-b f^{(\delta-\varepsilon)} & 0 & c-d f^{(\delta-\varepsilon)} \\
\bar{c}+\bar{d}\left(f^{(\varepsilon-\delta)}\right)^{*} & 0 & \bar{a}+\bar{b}\left(f^{(\varepsilon-\delta)}\right)^{*} & 0 \\
0 & \bar{c}-\bar{d}\left(f^{(\delta-\varepsilon)}\right)^{*} & 0 & \bar{a}-\bar{b}\left(f^{(\delta-\varepsilon)}\right)^{*}
\end{array}\right)
$$


is invertible on $l_{0}^{2} \oplus \ldots \oplus l_{0}^{2}$, and

(c) the singular integral operator

$$
\chi_{\Gamma_{\tau} \backslash \Gamma_{\tau, 1}}(a I+b S+(c I+d S) M) \chi_{\Gamma_{\tau} \backslash \Gamma_{\tau, 1}} I
$$

with $\Gamma_{\tau}:=e^{\mathrm{i} \omega_{\tau}}[0, \infty) \cup[0, \infty)$ and $\Gamma_{\tau, 1}:=e^{\mathrm{i} \omega_{\tau}}[0,1) \cup[0,1)$ is invertible on $L^{2}\left(\Gamma_{\tau} \backslash\right.$ $\left.\Gamma_{\tau, 1}, r_{\tau}\right) \subseteq L^{2}\left(\Gamma_{\tau}, r_{\tau}\right)$

Whereas it seems to be quite hard to analyse condition (c) further (it is actually equivalent to the invertibility of the Wiener-Hopf operator acting on the space $L^{2}\left([0, \infty), r_{\tau}\right) \oplus \ldots \oplus L^{2}\left([0, \infty), r_{\tau}\right)$ with generating function

$$
\left(\begin{array}{cccc}
a+b s & -b n_{2 \pi-\beta} & c+d s & -d n_{2 \pi-\beta} \\
b n_{\beta} & a-b s & d n_{\beta} & c-d s \\
\bar{c}-\bar{d} s & \bar{d} n_{\beta} & \bar{a}-\bar{b} s & \bar{b} n_{\beta} \\
-\bar{d} n_{2 \pi-\beta} & \bar{c}+\bar{d} s & -\bar{b} n_{2 \pi-\beta} & \bar{a}+\bar{b} s
\end{array}\right)
$$

where $\beta:=\omega_{\tau}$ and where $s(z):=\operatorname{coth} \pi\left(z+\mathrm{i}\left(1 / 2+r_{\tau}\right)\right)$ and $\left.n_{\beta}(z):=\frac{e^{\left(z+\mathrm{i}\left(1 / 2+r_{\tau}\right)\right)(\pi-\beta)}}{\sinh \pi\left(z+\mathrm{i}\left(1 / 2+r_{\tau}\right)\right)}\right)$, we have effective criteria to check the invertibility of the Toeplitz operator $T$ in condition (b).

Indeed, as in the proof of Lemma 3.3 one easily gets that the operator $T$ is invertible if and only if the Toeplitz operator with generating function

$$
\left(\begin{array}{cccc}
a+b f^{(\varepsilon-\delta)} & c+d f^{(\varepsilon-\delta)} & 0 & 0 \\
\bar{c}+\bar{d}\left(f^{(\varepsilon-\delta)}\right)^{*} & \bar{a}+\bar{b}\left(f^{(\varepsilon-\delta)}\right)^{*} & 0 & 0 \\
0 & 0 & a-b f^{(\delta-\varepsilon)} & c-d f^{(\delta-\varepsilon)} \\
0 & 0 & \bar{c}-\bar{d}\left(f^{(\delta-\varepsilon)}\right)^{*} & \bar{a}-\bar{b}\left(f^{(\delta-\varepsilon)}\right)^{*}
\end{array}\right)
$$

is invertible and, hence, if and only if the $2 \times 2$ block Toeplitz operators $T_{1}$ and $T_{2}$ with generating functions

$$
\left(\begin{array}{cc}
a+b f^{(\varepsilon-\delta)} & c+d f^{(\varepsilon-\delta)} \\
\bar{c}+\bar{d}\left(f^{(\varepsilon-\delta)}\right)^{*} & \bar{a}+\bar{b}\left(f^{(\varepsilon-\delta)}\right)^{*}
\end{array}\right), \quad\left(\begin{array}{cc}
a-b f^{(\delta-\varepsilon)} & c-d f^{(\delta-\varepsilon)} \\
\bar{c}-\bar{d}\left(f^{(\delta-\varepsilon)}\right)^{*} & \bar{a}-\bar{b}\left(f^{(\delta-\varepsilon)}\right)^{*}
\end{array}\right)
$$

respectively, are invertible.

Making use of the identity $\left(f^{(\nu)}\right)^{*}=-f^{(\nu)}$ (again from the proof of Lemma 3.3) we can rewrite the operator $T_{1}$ as

$$
\left(\begin{array}{ll}
a+b T\left(f^{(\varepsilon-\delta)}\right) & c+d T\left(f^{(\varepsilon-\delta)}\right) \\
\bar{c}-\bar{d} T\left(f^{(\varepsilon-\delta)}\right) & \bar{a}-\bar{b} T\left(f^{(\varepsilon-\delta)}\right)
\end{array}\right)
$$

and since all entries in this operator matrix commute with each other, we conclude that the operator $T_{1}$ is invertible if and only if the operator

$$
\begin{aligned}
& \left(a+b T\left(f^{(\varepsilon-\delta)}\right)\right)\left(\bar{a}-\bar{b} T\left(f^{(\varepsilon-\delta)}\right)\right)-\left(c+d T\left(f^{(\varepsilon-\delta)}\right)\right)\left(\bar{c}-\bar{d} T\left(f^{(\varepsilon-\delta)}\right)\right) \\
& =(a \bar{a}-c \bar{c}) I+(\bar{a} b-a \bar{b}-\bar{c} d+c \bar{d}) T\left(f^{(\varepsilon-\delta)}\right)-(b \bar{b}-d \bar{d})\left(T\left(f^{(\varepsilon-\delta)}\right)\right)^{2}
\end{aligned}
$$

is invertible. This clearly happens if and only if

$$
(a \bar{a}-c \bar{c}) I+(\bar{a} b-a \bar{b}-\bar{c} d+c \bar{d}) \lambda-(b \bar{b}-d \bar{d}) \lambda^{2} \neq 0
$$

for all $\lambda$ belonging to the spectrum of the Toeplitz operator $T\left(f^{(\varepsilon-\delta)}\right)$. 
So what we still need is the spectrum of that Toeplitz operator considered as acting on the Hilbert space $l^{2}$. For, abbreviate $\varepsilon-\delta$ by $\nu$ and observe that, for $\nu \neq 0$, the piecewise continuous function $f^{(\nu)}$ maps the unit circle into a circular $\operatorname{arc} \mathcal{C}_{\nu}$ in the complex plane which joins -1 to 1 and runs through the point $i \tan (\pi \nu / 2)$ (the center of that circular arc is $-\mathrm{i} \cot \pi \nu$, and its radius is equal to $1 /|\sin \pi \nu|)$. The well known criterion for the invertibility of Toeplitz operators with piecewise continuous generating function (see, e.g., Corollary 2.40 and Theorem 2.74 in [1]) states that the spectrum of $T\left(f^{(\nu)}\right)$ just coincides with that compact region in the complex plane which is bounded by the circular $\operatorname{arc} \mathcal{C}_{\nu}$ and the interval $[-1,1]$. Thus, denoting this region by $\mathcal{D}_{\nu}$, we get

Lemma 5.3. The Toeplitz operator $T_{1}$ is invertible if and only if (5.3) is satisfied for all $\lambda \in \mathcal{D}_{\varepsilon-\delta}$.

An analogous result holds for the invertibility of $T_{2}$.

\section{REFERENCES}

[1] A. Böttcher and B. Silbermann, Analysis of Toeplitz Operators, Akademie-Verlag, Berlin, 1990.

[2] M. CostaBel, Boundary integral operators on curved polygons, Ann. Math. Pure Appl., 133 (1983), pp. 305-326.

[3] M. Costabel and E. P. Stephan, The method of Mellin transformation for boundary integral equations on curves with corners, in Numerical Solution of Singular Integral Equations, A. Gerasoulis and R. Vichnevetsky, ed., Proc. IMACS Int. Symp. Lehign. Univ. Bethlehem, Pennsylvania, U.S.A., 1984, pp. 95-102.

[4] M. Costabel AND E. P. Stephan, Boundary integral equations for mixed boundary value problems in polygonal domains and Galerkin approximations, in Banach Center Publications 15, PWN, Warsaw, 1985, pp. 175-251.

[5] M. Costabel And E. P. Stephan, On the convergence of collocation methods for boundary integral equations on polygons, Math. Comp., 49 (1987), pp. 461-678.

[6] V. D. DidenKo AND V. N. MATSKUL, On the approximate solution of singular integral equations with conjugation, Zh. Vychisl. Mat. i Mat. Fiz., 29:3 (1989), pp. 392-404 (Russian, English transl. in USSR Comput. Math. and Math. Phys., 29 (1989), pp. 45-54).

[7] V. D. Didenko And B. Silbermann, On the stability of some operator sequences and the approximate solution of singular integral equations with conjugation, Integral Equations Operator Theory, 16 (1993), pp. 224-243.

[8] V. D. Didenko and E.Venturino, Approximate solution of some Mellin equations with conjugation, Integral Equations Operator Theory, 25 (1996), pp. 163-184.

[9] V. D. Didenko, S. Roch, AND B. Silbermann, Approximation methods for singular integral equations with conjugation on curves with corners, SIAM J. Numer. Anal., 32:6 (1995), pp. 1910-1939.

[10] R. V. Duduchava, On general singular integral operators of the plane theory of elasticity, Rend. Politechn. Torino, 42:3 (1984), pp. 15-41.

[11] R. V. Duduchava, General singular integral equations and basic problems of the plane theory of elasticity, Trudy Tbilissk. Matem. Inst., 82 (1986), pp. 45-89 (Russian).

[12] I. G. Graham and G. A. Chandler, High-order methods for linear functionals of solutions of second kind integral equations, SIAM J. Num. Anal., 25 (1988), pp. 1118-1179.

[13] I. C. Gohberg AND N. YA. KRUPNiK, On the algebra generated by Toeplitz matrices, Funk. Analiz i ego Prilozh., 3:2 (1969), pp. 46-56 (Russian).

[14] R. Hagen, S. Roch, ANd B. Silbermann, Spectral Theory of Approximation Methods for Convolution Operators, Birkhäuser Verlag, Basel, Boston, Berlin, 1995.

[15] A. N. KalandiYa, Mathematical Methods of Two-dimensional Elasticity, Nauka, Moscow, 1973 (Russian), and Mir Publishers, Moscow, 1975.

[16] N. I. Muskhelishvili, Some Basic Problems of the Mathematical Theory of Elasticity, Nauka, Moscow, 1966 (Russian), and Nordhoff, Groningen, 1953.

[17] N. I. MuskHELishvili, Singular Integral Equations, Nauka, Moscow, 1968 (Russian), and Nordhoff, Groningen, 1963. 
[18] V. V. Panasuk, M. P. Savruk, AND A. P. Dazishin, On the stress distribution in the neighborhood of cracks in plates and membranes, Naukowa Dumka, Kiev, 1976 (Russian).

[19] S. PRÖSSDORF AND A. RATHSFELD, Quadrature and collocation methods for singular integral equations on curves with corners, ZfAA, 8 (1989), pp. 197-220.

[20] S. PRÖSSDORF AND A. RATHSFELd, Mellin techniques in the numerical analysis, Report RMath-06/88, Karl-Weierstrass-Institut für Mathematik, Berlin, 1988.

[21] S. Prössdorf And B. Silbermann, Numerical Analysis for Integral and Related Operator Equations, Akademie-Verlag, Berlin, 1991 and Birkhäuser Verlag, Basel, Boston, Stuttgart, 1991.

[22] B. Silbermann, Lokale Theorie des Reduktionsverfahrens fr Toeplitzoperatoren, Math. Nachr., 104 (1981), pp. 137-146.

[23] I. N. VeKuA, Generalized Analytic Functions, Nauka, Moscow, 1988 (Russian). 
